\title{
Il diritto di denunciare la mancanza di idoneità dei titolari degli uffici ecclesiastici*
}

\section{The Right to Denounce the Lack of Suitability of Holders of Ecclesiastical Office}

\author{
Geraldina BONI \\ Professoressa Ordinaria Dipartimento di Scienze Giuridiche \\ Università di Bologna. Bologna \\ orcid 0000-0003-2098-8469 \\ geraldina.boni@unibo.it
}

Resume: Quale ulteriore progresso nella formalizzazione tecnico-giuridica della natura diaconale di servizio del governo ecclesiastico e quindi per consolidare il diritto-dovere al buon governo nella Chiesa, nel presente studio proponiamo de iure condendo il riconoscimento esplicito del diritto-dovere dei fedeli di attivarsi in prima persona 'denunciando' la mancanza di idoneità dei titolari di uffici ecclesiastici: e questo sia durante la fase previa di designazione, con opportuni interventi nel procedimento amministrativo di nomina, sia soprattutto una volta che la prowista sia awenuta, quale reazione nei confronti della sua non congruità. L'itinerario giuridico per fondare siffatta pretesa, articolata a più livelli, è complesso e per compierlo abbiamo esaminato in via euristica vari istituti giuridici contemplati dal diritto canonico: si tratta di una sfida da affrontare e che non può più essere differita.

Parole chiave: Denuncia, Codex luris Canonici, Ufficio ecclesiastico, Idoneità, Corresponsabilità, Abuso.
Abstract: Given further legal formalization of the diaconal nature of ecclesiastical governance and to consolidate the right of the ecclesial community to good governance, this study sets out from a de iure condendo perspective the explicit recognition of the right and duty of all Catholic people to play an active role in 'denouncing' the lack of suitability of holders of ecclesiastical office: either in the stage prior to appointment, by intervening in the administrative phase of the nomination process, or more importantly after the appointment has been made, should there be any incongruity between the office-holder and the post. The legal context to such a claim is complex and a large number of juridical aspects of canon law have been studied in order to address it; the issue is a challenging one but it can no longer be deferred.

Keywords: Complaint, Codex luris Canonici, Ecclesiastical Office, Eligibility, Co-Responsibility, Abuse.

* Sintetizziamo in questo saggio le argomentazioni più ampiamente e approfonditamente sviluppate nella monografia G. BONI, Il buon governo nella Chiesa. Inidoneità agli uffici e denuncia dei fedeli, Mucchi Editore, Modena 2019, alla quale rinviamo per l'apparato di note chiarificatrici e per ulteriori riferimenti bibliografici. 
SOMMARIO: 1. Il diritto-dovere dei christifideles al buon governo e, pertanto, a buoni governanti. 2. I fedeli e la provvista degli uffici ecclesiastici. Il margine di discrezionalità dell'autorità e la sententia dei fedeli (can. $212 \$ 3)$. 3. L“esercizio inidoneo' dell'ufficio. Alcuni suggerimenti desumibili dalla disciplina del pubblico impiego in Italia. 4. Dal diritto di petizione dei fedeli (can. $212 \$ 2$ ) alla rivendicazione di un interesse diffuso. 5. Dall'obbligo di rivelare impedimenti al matrimonio e ai sacri ordini... 6. ...fino alle suggestioni discendenti dall'analogia con le azioni popolari: una proposta de iure condendo. 7. Qualche riflessione sulla denuntiatio christifidelium in ordine alle fattispecie di cui al can. 1389 e in altri casi. Una 'direzione di marcia' desumibile da alcuni provvedimenti. 8. Dignus est!'

\section{IL DIRITTO-DOVERE DEI CHRISTIFIDELES AL BUON GOVERNO E, PERTANTO, A BUONI GOVERNANTI}

N on sarà mai sottolineata a sufficienza la portata epocale delle affermazioni del Concilio Vaticano II, lapidarie nella loro nettezza, secondo le quali «L'ufficio che il Signore affidò ai pastori del suo popolo è un vero servizio, che nella sacra Scrittura è chiamato significativamente 'diaconia', cioè ministero (cfr. At 1,17 e 25; 21,19; Rm 11,13; $1 \mathrm{Tm}$ 1,12)» (Costituzione dogmatica Lumen gentium, n. 24). Affermazioni echeggiate senza posa e senza alcuna soluzione di continuità anche dagli ultimi successori di Pietro. D'altronde, se è vero che si tratta di principio cardinale ascrivibile all'ontologia, all'essere della Chiesa, deducibile con lampante chiarezza dalla predicazione del Figlio dell'uomo, venuto non per essere servito ma per servire (Mt 20,2428; Mc 10,42-45; Lc 22,25-26), reiteratamente ribadito nel corso dei secoli e sin dagli albori dalle menti più illuminate, tuttavia esso altrettanto reiteratamente è stato ignorato se non tradito: e soprattutto numerose delle sue potenziali implicazioni attendono ancora di essere implementate, nonostante le innovazioni introdotte nello ius Ecclesiae - segnatamente nelle codificazioni proprio sul solco dell'assise novecentesca. Pur essendo gli uffici nella Chiesa di differente natura, per quanto qui interessa argomentare si terranno in precipua considerazione, tra quelli che attengono al triplice munus di Cristo, gli officia propriamente afferenti alla funzione di governare la compagine ecclesiale, sia pure in policroma configurazione e in 'frazioni' più o meno consistenti della potestas iurisdictionis ${ }^{1}$. E al riguardo, muovendo appunto dalla pre-

${ }^{1}$ Cfr., per tutti, le precisazioni di A. VIANA, Aspectos de la relación del fiel con la organización eclesiástica, Fidelium iura 4 (1994) 79-106. 
messa assiologica con cui abbiamo esordito - dunque, al fondo, dallo ius divinum, e quindi proprio per declinare meglio nel diritto positivo tale imperativo - sempre più risolutamente, e già dalla fine degli anni Sessanta ${ }^{2}$, la canonistica ha mirato a congetturare ed ingegnerizzare un dovere dell'autorità e specularmente un diritto del fedeli al buon governo nella Chiesa ${ }^{3}$, malgrado la sua assenza dall'elenco stilato nella codificazione post-conciliare ${ }^{4}$ : il quale comunque, unanimemente si concorda, non è esaustivo. In questa sede ci limiteremo ad esplorarne un aspetto apparentemente secondario ed invece dirimente per poter delineare quale vero diritto quello de quo: un aspetto invero sinora per nulla o quasi investigato. Avventurandoci quindi su lidi ancora vergini, si tenterà di rispondere al quesito se i cbristifideles dispongano nello ius canonicum della possibilità e quindi di strumenti per reagire all'insediamento in uffici ecclesiastici, ma pure - espandendo il diametro dello scandaglio - in $m u$ nera, ministeria e qualunque servizio nella Chiesa, di persone inidonee, non fornite delle qualità necessarie $\mathrm{o}$ inette, $\mathrm{e}$ che perciò si può presumere, con un indice di approssimazione notevole, mal adempiranno al loro compito: ripercuotendosi questa 'infedeltà al servizio' in una lesione del buon governo e pertanto del bonum commune, con strascichi, non va mai sottaciuto, non di rado esiziali sulla salus animarum di coloro che ne risentono. Anzitutto, dunque, se essi godano del diritto-dovere (come vedremo) di fare presente, affinché venga sanata, tale inidoneità, quindi di 'denunciarla': assumendo ora tale dicitura in senso generico, rinviando al prosieguo per ulteriori distinguo. Rischierebbe infatti di trasformarsi in un flatus vocis od anche in un'accondiscendenza ai fedeli solo di facciata e paternalistica il dipingere con dovizia di dettagli un di-

\footnotetext{
2 Cfr. J. Hervada - P. Lombardía, El derecho del pueblo de Dios. Hacia un sistema de derecho canónico, I, Introducción. La constitución de la Iglesia, Universidad de Navarra, Pamplona 1970, 309; J. HeRvaDA, Elementos de derecho constitucional canónico, Eunsa, Pamplona 1987, 146; si veda anche J. HerRanZ, De principio legalitatis in exercicio potestatis ecclesiasticae, in Pontificia Commissio Codici IURIS Canonici Recognoscendo (ed.), Acta Conventus Internationalis Canonistarum Romae diebus 20-25 maii 1968 celebrati, Typis Polyglottis Vaticanis, Città del Vaticano 1970, 224; P. VILADRICH, La declaración de derechos y deberes de los fieles (Cánones 10 al 30), in REDACCIÓN IUS CANONICUM (ed.), El proyecto de Ley fundamental de la Iglesia. Texto y análisis crítico, Eunsa, Pamplona 1971, 158; Á. DeL PorTILlo, Laici e fedeli nella Chiesa. Le basi dei loro statuti giuridici, Giuffrè Editore, Milano 1999, 64-74 e 125-127 (prima edizione Fieles y laicos en la Iglesia. Bases de sus respectivos estatutos jurídicos, Eunsa, Pamplona 1981).

3 Cfr. da ultimo M. Del Pozzo, Lo statuto giuridico fondamentale del fedele, Edusc, Roma 2018, 162-163.

${ }^{4}$ In questa trattazione, laddove non si specifichi altrimenti, parlando di Codice e canoni ci riferiamo sempre al vigente Codex Iuris Canonici per la Chiesa latina.
} 
ritto che però non si può rivendicare e far valere laddove pesantemente minacciato in alcuni delicati e determinanti decorsi decisionali: come proprio quello della provvisione degli uffici ecclesiastici, adoperando anche qui il termine in senso generale e complessivo come conferimento dell'incarico a persona previamente designata e riconosciuta idonea.

Non è superfetazione avvertire che il diritto al buon governo, secondo la configurazione comune a tutti i diritti nella Chiesa ed anzi forse più patentemente di altri, deve essere improntato ed anche trasfigurato dall'afflato comunionale. Esso non va, cioè, rivendicato faziosamente contra l'autorità ecclesiastica per perseguire un interesse proprio ed esclusivo in assurda dissociazione, quasi in una rivalsa ecclesialmente contraddittoria o addirittura in un'opposizione tra persona e istituzione: per converso, che gli uffici nella Chiesa siano ricoperti da persone rette, preparate ed efficienti è un valore da presidiarsi da parte di tutti i componenti del popolo di Dio a pro della communio. Anzi è specialmente la logica che innerva quest'ultima ad imporre che la reazione avverso una nomina pregiudizievole non dovrebbe attivarsi unicamente allorquando si sia compromessa o anche solo lambita la propria 'spettanza'. Al contrario, a prescindere dalla medesima e quindi da un nocumento individuale, qualunque fedele, e proprio in forza della sua basica condizione battesimale, dovrebbe essere tenuto all'iniziativa laddove la componente gerarchica manchi nella realizzazione della missione che è stata affidata all'Ecclesia: all'Ecclesia tutta, ministri e semplici battezzati, sia pur nella diversità di condizioni e mansioni.

Il buon governo sul quale si dispiega l'ipotizzato diritto dei fedeli parrebbe prima facie un concetto astratto e impalpabile: esso però, passando attraverso apparati organizzativi, unità operative, appunto officia, ministeria e munera, al fondo e in sostanza si impernia e si alimenta di persone fisiche agenti bic et nunc. Pertanto si materializza, si incarna cioè, e non può non incarnarsi, in buoni governanti ed esecutori: senza una loro tempra ben forgiata il buon governo è, metaforicamente, una macchina senza carburante, e dalla qualità delle persone dipende la qualità delle decisioni. Risalta dunque icasticamente la spina dorsale cruciale della cernita e investitura di coloro che saranno incaricati, nella Chiesa pellegrina, di 'sovrintendere' in vario modo e con varie incombenze al popolo di Dio, ovvero che riceveranno i differenti officia, munera e ministeria e che devono possedere le specifiche e sfaccettate qualità sagomate su ogni ruolo ecclesiale: e questo vale sia che la scelta ricada su un chierico o un consacrato (i quali pure dovrebbero già essere stati sotto- 
posti ad un previo 'test attitudinale', per così dire, per lo stato di vita prescelto ${ }^{5}$ ), ovvero su laici, uomini e donne senza discriminazione, qui idonei reperiantur (can. 228). Ciò è tanto importante che proprio in questo discernimento e responsabilità è stato ravvisato «il baricentro della c.d. potestas (o della 'giurisdizione' in senso ampio)» ${ }^{6}$.

\section{I FEDELI E LA PROVVISTA DEGLI UFFICI ECCLESIASTICI. \\ IL MARGINE DI DISCREZIONALITÀ DELL'AUTORITÀ \\ E LA SENTENTIA DEI FEDELI (CAN. $212 \$ 3$ )}

$\grave{E}$ dunque riguardo allo snodo cruciale appena individuato che ci si deve domandare se i fedeli - anche se prevalentemente, come emergerà dal nostro argomentare, alludiamo ai laici - abbiano o no 'voce in capitolo' dinanzi a colui al quale tale atto è addebitabile. Se si conviene infatti sull'assunto difficilmente controvertibile secondo cui in Ecclesia «il buon governo non è il frutto del genio o del carisma personale dell'ufficio capitale ma della formazione e collaborazione di tutte le forze vive e vitali della comunità credente» ${ }^{7}$, e che solo da questa simbiosi e compenetrazione di più voci può orchestrarsi una coralità armonica, da ciò non possono che profluire un plesso di corollari anche in relazione al tema ora in esame.

In primis ne dovrebbe discendere il permesso di visionare e verificare in qualche modo l'intera procedura che culmina nel conferimento dell'ufficio, quale atto sì libero dell'autore ma che si innesta peraltro in una serie di passaggi almeno tendenzialmente disciplinati. Nel senso che è indubbiamente vero come l'incremento della legalità e della procedimentalizzazione - sempre moderata e non elefantiaca, distante cioè da talune derive secolari - anche nella collazione degli uffici non siano per nulla sicura malleveria di identificare persone rette e preparate: ciononostante seguire, senza mitizzazioni appunto, certi binari procedurali non ha controindicazioni, tutt'altro, soprattutto al fine di rendere la provvisione decifrabile e cristallina per l'esterno, cioè per la comunità. E questo vale anche per quegli atti i quali non possono che conservare un

${ }^{5}$ Cfr., per tutti, A. VIANA, La comprobación de la idoneidad para el oficio eclesiástico y el orden sagrado, Ius Ecclesiae 28 (2016) 345-366.

${ }^{6}$ P. GHERRI, L'autotutela amministrativa come supplemento di conoscenza: la remonstratio canonica (Cann. 1732-1734 CIC), in IDEM (ed.), Decidere e giudicare nella Chiesa. Atti della VI giornata canonistica interdisciplinare, Lateran University Press, Città del Vaticano 2012, 321.

${ }^{7}$ M. Del Pozzo, Lo statuto giuridico..., cit., 163. 
vasto raggio di discrezionalità dell'autorità, come inevitabilmente e sovente la provvista degli uffici: ciò che rende più spinoso sindacarli e censurarli. Ma l'insindacabilità totale e l'immunità da ogni 'ispezione' segnatamente comunitaria non è accettabile, a pena di mutare e far sfociare la discrezionalità, tale maior libertas, in arbitrio: qualsiasi atto discrezionale deve essere comunque 'giusto'.

Nella lacunosità e laconicità della codificazione relativamente al procedimento amministrativo, aspramente lamentate, certo va ottemperato al can. 50 secondo il cui dettato (invero anch'esso stringato e scheletrico) prima di emettere un decreto singolare - ma la norma ha portata più larga - l'autorità deve propedeuticamente ricercare «necessarias notitias et probationes» e, per quanto possibile, ascoltare coloro i cui diritti possono essere lesi. Al riguardo va condivisa la posizione di chi sostiene che «la sensibilidad que requiere el sometimiento de la actividad de la Administración a esta norma debería plasmarse [...] en una interpretación estricta del "quantum fieri potest" y en una interpretación amplia del "quorum iura laedi possint" $\rangle^{8}$ : sì che in particolare l'ascolto, da omettersi solo sporadicamente, non debba perimetrarsi alle persone titolari di un diritto in senso stretto che possa essere effettivamente leso, ma concerna coloro che possano offrire in qualche modo un contributo giovevole, potendo «le loro dichiarazioni [...] apportare dati di conoscenza e aspettative legittime in relazione ai beni che entrano in gioco nel procedimento» ${ }^{9}$.

Perché poi i fedeli possano essere chiamati ad un controllo avvertito si deve anzitutto consolidare il dovere dell'autorità ecclesiastica di informare non solo sull'avvio del procedimento che condurrà alle provviste, ma anche sugli screenings delle persone che solitamente le precedono, divulgando senza remore i curricula dei prescelti: la pertinenza delle notizie unicamente al compimento della mansione demandata non dovrebbe creare alcun problema di violazione della buona fama e dell'intimità di cui al can. 220. Ci sembra, per converso, che questo sarebbe un segno istruttivo che ammaestrerebbe circa la volontà ecclesiale di affossare definitivamente l'indulgere al riserbo ed alla segretezza anche laddove non indispensabili, per aprirsi a quella tersa limpidità tanto bramata da papa Francesco. È giunto il momento di recepire nell'ordinamento canonico quanto è stato già auspicato da tempo de lege ferenda dalla

${ }^{8}$ J. Miras, Sub can. 50, in Á. Marzoa - J. Miras - R. Rodríguez-Ocaña (eds.), Comentario exegético al Código de Derecho Canónico, I, tercera edición actualizada, Eunsa, Pamplona 2002, 561.

9 J. Miras - J. Canosa - E. Baura, Compendio di diritto amministrativo canonico, Edusc, Roma 2007, 181 . 
dottrina nel senso di una sanzione esplicita del diritto all'informazione nella Chiesa e sulla Chiesa (un'informazione cioè pertinente, coerente e aderente alla medesima): un riconoscimento non più prorogabile e posticipabile oggi nell'era informatica e digitale in cui nulla può tenersi celato, ma certo - e a maggior ragione proprio per queste perigliose evoluzioni - da adattare alla specificità della realitas ecclesiale non eguagliabile agli ordinamenti democratici. Ovviamente «Non si tratta di rendere patente tutto (una sorta di conduzione "a porte aperte") ma di manifestare quanto riguarda il possibile intervento della collettività o un qualificato interesse personale» ${ }^{10}$. Se i fedeli, però, sono tenuti all'oscuro ovvero sono informati in maniera astrusa o ermetica, ovvero anche incompleta ed edulcorata inevitabilmente essi sono «messi sin dall'inizio "fuori gioco"» ${ }^{11}$. La corresponsabilità dei fedeli quanto al governo si realizzerebbe qui non con una diretta 'immissione' nel medesimo, ma attraverso la preoccupazione a che esso rimanga teleologicamente sempre orientato al bonum commune, sia quindi davvero buon governo a cui tutti i fedeli, senza ostracismi, si possono e devono sentire interpellati. Certo «la partecipazione dei fedeli al governo non può degenerare in una valanga di rivendicazioni, di pareri e di consultazioni erga omnia che trasformerebbe il governo ecclesiale in una specie di "percorso di guerra" $\gg^{12}$ : eppure l'apprensione preconcetta verso tali possibili - tra l'altro arginabili - intemperanze non può essere paralizzante per un diritto che, in caso contrario, si rivelerebbe solo un diafano fantasma e che invece non può non avere un futuro nella comunione ecclesiale.

Indubbiamente, inoltre, i titolari degli uffici ecclesiastici non devono essere apprezzati solo per le capacità di gestire adempimenti giuridici, burocratici, economici, non sono anzitutto professionisti e dirigenti, managers e imprenditori: ça va sans dire, senza dover ogni volta rimembrare - invero a volte un poco ad pompam - la logica misterica, teologica, soprannaturale e trascendente del governo ecclesiastico, che non è in questione e nessuno vuole appannare, o, peggio, ricusare. Ma la Chiesa, predica senza sosta papa Bergoglio, deve vagheggiare indefessamente lo sviluppo umano integrale. Così le virtù teologali devono risplendere nel governante in modo che il suo sguardo sia sempre calamitato verso il giudizio divino e la salvezza delle anime. Per certi

\footnotetext{
10 M. DEL Pozzo, Lo statuto giuridico..., cit., 148.

11 J.-P. SCHOUPPE, Diritti fondamentali dei fedeli in rapporto alla partecipazione al governo dei beni temporali, Ius Ecclesiae 26 (2014) 411.

12 J.-P. Schouppe, Diritti fondamentali..., cit., 400.
} 
munera, poi, serve equilibrio mentale ed equanimità, sobrietà e ascetico distacco rispetto ai beni temporali, spirito di sacrificio, obbedienza, sentimento comunitario, ecc.: senza contare i requisiti più spirituali per essere ammessi al seminario o al noviziato; ovvero ancora l'accertamento della non incrinatura della comunione (comunione tra l'altro richiesta in 'gradi' diversi a seconda degli uffici ${ }^{13}$ ). D'altronde, però, anche le attitudini meno 'soprannaturali' e più 'profane', ovvero più 'intellettuali' che 'pastorali', ma che rendono governanti competenti e ferrati, dinamici e tempestivi, insomma prudenti ed efficienti, sono 'talenti' che non alterano né avviliscono la sostanza e la finalizzazione, sempre invero soteriologica ed escatologica, dell'ufficio ecclesiale. Tutto all'opposto, solo una visione unitaria e sintonica dell'aspetto pastorale e di quello pratico-amministrativo può essere foriera di un servizio proficuo: si è chiosato al riguardo che «la dimensione soprannaturale non supplisce le virtù umane, ma al contrario le presuppone» ${ }^{14}$. Tali virtù umane, e qui segnatamente ci riferiamo alle competenze richieste che vanno a 'riempire' direttamente l'idoneità (le competenze quindi insurrogabili, non quelle che rendono un candidato più idoneo in una rosa di soggetti che le detengono e nella scelta dei quali può dispiegarsi appieno la discrezionalità dell'autorità, sovente minutamente edotta di ogni circostanza personale), forse si potrebbero, almeno parzialmente, cioè per alcuni contenuti sostanziali - lo proponiamo quale ipotesi di lavoro da sottoporre a verifica - ricondurre a quei 'concetti giuridici indeterminati' di cui non da oggi disquisisce la dottrina. Essi sono presupposti di fatto di una deliberazione comportanti una valutazione da effettuarsi da parte dall'autorità ecclesiastica in qualche modo oggettivabile attraverso conoscenze di varia tipologia: valutazione pertanto sindacabile e che gli stessi fedeli possono vagliare, eventualmente respingendo il giudizio finale cui si è pervenuti. L'apprensione infatti di tali concetti, enunciati ma non definiti in modo tassativo dall'ordinamento, è pilotata dall'utilizzo di parametri precisi, sì che in certa misura si 'contiene' la discrezionalità, non potendo l'autorità

${ }^{13}$ Cfr. J. I. ARRIETA, El sistema canónico de selección y de provisión de cargos, relazione al XIII Simposio Internacional del Instituto Martín De Azpilcueta de la Universidad de Navarra Las relaciones de servicio según el derecho canónico y estatal. Oficios, idoneidad, relaciones laborales, svoltosi a Pamplona dal 7 al 9 novembre 2018, p. 13 (con riferimento alle pagine del dattiloscritto della prima e provvisoria redazione del testo).

${ }^{14}$ E. BAURA, Il "buon governo": diritti e doveri dei fedeli e dei pastori, in GRUPPO ITALIANO DocenTI Di DiRITTO CANONICO (a cura di), Il governo nel servizio della comunione ecclesiale, Glossa, Milano 2017, 29. 
discostarsi dai medesimi: e se dovesse farlo, divaricandosi irragionevolmente in questo momento conoscitivo dai criteri tecnici da applicarsi, potrebbe e dovrebbe essere ripresa, 'denunciandosi' appunto l'assenza di idoneità del nominato, e, dunque, facendo franare l'opportunità della provvista. Anche se a volte - deve ammettersi - è davvero impervio districarsi tra oggettività e soggettivismo della valutazione.

Comunque sia, quanto all'idoneità agli uffici, è forse proprio su quest'ultimo versante - anche se non esclusivamente - che la sententia, la quale il canone $212 \$ 3$ i fedeli, e peculiarmente i laici, in modo proporzionato alla scienza, alla competenza e al prestigio di cui godono, hanno il diritto e anzi talvolta anche il dovere di manifestare ai sacri pastori in merito a ciò che riguarda il bene della Chiesa, può rinvenire la superficie privilegiata sulla quale sperimentarsi. Non perché i 'semplici' fedeli non possano mai pronunciarsi sulla genuinità della vocazione e sull'autenticità degli intenti (ovviamente sempre non in via risolutoria): ma perché, mentre tale discernimento meglio si addice al carisma ed all'inclinazione 'paterna' dei superiores (non in quanto tali ma in virtù delle loro funzioni), l'apprezzamento viceversa di attitudini più professionali e 'secolari' presume un maturo possesso delle medesime che può forse difettare alle autorità ecclesiastiche, rendendo più stringente il dovere del fedele e specialmente del laico, «particolarmente qualificato per la sua esperienza in rebus bumanis ${ }^{15}$. Del pari si potrebbe dall'esterno - ma sempre all'interno del popolo di Dio - condannare il fatto che, da parte dell'autorità, si siano omesse le verifiche necessarie, eludendo quell'itinerario di raccolta documentata di informazioni che solo può essere presidio di una scelta felice. In sostanza il fedele, anche non consultato, di sua iniziativa trasmette all'autorità un suo 'pensiero' (secondo l'invalsa versione italiana del can. $212 \$ 3$ del Codex Iuris Canonici), 'un fatto' di cui ha contezza affinché essa sopperisca a preservazione del buon governo: presentando una «semplice denuncia» ${ }^{16}$, una dichiarazione di conoscenza (mentre il ricorso e l'azione sono eminentemente dichiarazioni di volontà) per premere sull'autorità ad agire d'ufficio. Un canone, il $212 \$ 3$ - tra l'altro matrice di quella norma la quale apre alla partecipazione comunitaria la 'confezione' del decreto - che è davvero uno dei segnacoli più vividi del 'new deal' inaugurato dal Vaticano II, eppure ancora non abbastanza valorizzato nell'ordinamento canonico: benché non brilli nella

${ }^{15}$ I. ZUANAZZI, La corresponsabilità dei fedeli laici nel governo ecclesiale, in Il governo nel servizio..., cit., 133.

${ }^{16}$ E. LABANDEIRA, Il ricorso gerarchico canonico: «petitum» e «causa petendi», Ius Ecclesiae 3 (1991) 104. 
sua redazione testuale, specie per quella pedante e pleonastica ripetizione delle limitazioni che dovrebbero perimetrare l'esternazione del pensiero da parte dei christifideles, da cui ancora traspira una qualche prevenzione e diffidenza che invece il Concilio del Novecento aveva serenamente abbandonato. Tanto che i fedeli (tutti e non una oligarchia) sono incitati a manifestare la loro opinione: certo con l'attenzione mai deposta a non propalare opinioni erronee, irriverenti, insolenti, ingiuriose, segnatamente laddove si possa profanare la dignità delle persone. E ciascuno di essi pondera pro scientia, competentia et praestantia di cui gode: requisiti di non facile apprezzamento e che tuttavia non devono condurre a pregiudiziali emarginazioni da parte dell'autorità, se non altro accordando il beneficio del dubbio (can. 18) per non riservare indebitamente ad una minoranza quel diritto che spetta a tutti in virtù del solo battesimo. Se quindi il fedele, in base alle informazioni di cui dispone (anche per la sua condizione personale), si persuade profondamente in buona fede che 'siederà' o già 'sieda' a ricoprire una carica qualcuno sprovvisto dell'idoneità e che dunque influirà negativamente a compromissione del bene comune, ha il diritto, anzi diremmo per il bene della Chiesa il dovere, senza piaggeria né alcun timore reverenziale ma con schiettezza - e anche senza particolari formalità -, di notificarlo all'autorità ecclesiastica: «In altre parole, il diritto di "opinione" comprende anche quello di denuncia, al quale corrisponde il dovere dell'autorità di attivarsi per risolvere la questione denunciata ed informare il denunciante del risultato o del giudizio dell'autorità sul tema in questione. Lasciar cadere la denuncia in un "silenzio assordante" non è certo compatibile con il buon governo» ${ }^{17}$. Per gli esempi, numerosi, alcuni anche di impellente attualità, non possiamo ora che rinviare alla versione più estesa del nostro studio, pubblicato in forma monografica ${ }^{18}$.

\section{L'ESERCIZIO INIDONEO' DELL'UFFICIO. ALCUNI SUGGERIMENTI DESUMIBILI DALLA DISCIPLINA DEL PUBBLICO IMPIEGO IN ITALIA}

Potrebbe poi accadere che il titolare di un ufficio rifiuti ostinatamente di 'aggiornarsi', rigetti di sforzarsi per impadronirsi di abilità indispensabili al fine di svolgere proficuamente il suo incarico. $\mathrm{O}$ comunque potrebbe verificarsi che, per l'ineliminabile fallibilità umana ed altresì per le tentazioni che

17 E. BAURA, Il "buon governo"..., cit., 20.

${ }^{18}$ Cfr. G. BonI, Il buon governo nella Chiesa..., cit. 
possono sempre contaminare l'esercizio del potere, non dimostri abbastanza probità o incorruttibilità, rivelando la sua inidoneità proprio con l'esercizio inidoneo' dell'ufficio stesso: «Quien fue apto para un determinado oficio puede dejar de serlo, como consecuencia de los ataques del mundo, el demonio y la carne. La semilla puede caer en terreno pedregoso o crecer entre espinos y ser ahogada (Mt 13,21 y 22). El sistema de la remoción administrativa del titular del oficio o incluso la privación penal del cargo, son instrumentos del derecho canónico para reaccionar ante esas situaciones de inaptitud sucedida con posterioridad al nombramiento» ${ }^{19}$. Tali condotte non possono restare senza contraccolpi: d'altro canto se la Chiesa biasima le autorità secolari che non controllano e castigano rapidamente i propri 'funzionari', ciò che vale ad extra dovrebbe, per non essere malinteso, valere - e con maggior durezza - anche ad intra. L'autorità ecclesiastica non può cioè, una volta effettuata la provvista, rinunciare o abdicare al suo dovere indeclinabile di soprintendere alacremente: «Nel proseguo del rapporto di servizio, invece, sarà rilevante lo svolgimento effettivo delle funzioni nonché la sua incidenza nella comunità. Questo svolgimento è oggetto di uno specifico dovere di vigilanza da parte dell'autorità, avente come scopo primario la protezione dei diritti dei soggetti cui riguardano le funzioni, che sono la comunità e i titolari stessi degli uffici. La stessa autorità gode anche di uno spazio di libera decisione per prendere in considerazione - specialmente in quegli uffici in cui la fiducia gioca un ruolo di primo ordine e in quegli altri di natura predominantemente tecnica -, la qualità del servizio prestato, con l'eventuale capacità di modificare il rapporto o di concluderlo, secondo le condizioni stabilite nella legge e nella provvista dell'ufficio ${ }^{20}$. E anche in questo dovere di vigilanza non deve essere obnubilato l'aiuto che può essere somministrato dai fedeli 'denunciando' mende, deficienze, inadeguatezze.

È noto che assai più che funzionari delinquenti e scellerati, sono specialmente dipendenti oziosi, assenteisti, e, appunto, incompetenti, dunque inidonei, a costituire un fardello onerosissimo di cui tutte le pubbliche amministrazioni sono gravate: a discapito dei cittadini che ne sono le vittime spesso indifese. In Italia, ad esempio, soprattutto a partire dagli anni Novanta del secolo appena congedato si è assistito ad una proliferazione di riforme legislative volte a migliorare l'efficienza amministrativa che dipende primariamente

\footnotetext{
19 A. VIANA, La comprobación de la idoneidad..., cit., 347.

20 F. PUIG, Governo e discrezionalità nella collazione di uffici ecclesiastici, Ius Ecclesiae 30 (2018) 58.
} 
dai funzionari. Così non si è tentennato a perorare un'emulazione al riguardo da parte dell'ordinamento canonico, patrocinando: «Mi pare che l'efficienza richiesta nella società civile dovrebbe essere presa come punto di riferimento per determinare il buon governo nella Chiesa $»^{21}$; ed anche: $« \mathrm{Ci}$ si potrebbe chiedere se non fosse possibile mutuare dall'ambito civile la pratica di generare standard di diligenza, configurati come livelli di prestazione esigibile, valutabili in sede di governo e in certi casi in sede di ricorso, vale a dire capaci di creare delle responsabilità per inadempimento» ${ }^{22}$. Al fine di prevenire e schivare gli strali facilmente pronosticabili da parte di chi eccepirà l'errore anzitutto epistemologico di un'acritica mimesi degli ordinamenti secolari, ancora una volta ribadiamo che il trapianto in Ecclesia non potrà mai essere eseguito senza un'adeguata profilassi e immunizzazione, poiché il rischio di rigetto è sempre in agguato.

Con questa accortezza, assumendo a specimen l'ordinamento italiano, un punto di riferimento potrebbe essere costituito dal modello (invero assunto in molti altri ordinamenti, oltre che 'sponsorizzato' a livello sovranazionale ${ }^{23}$ ) dei codici di comportamento dei dipendenti pubblici (codice generale nazionale e codici interni per ciascuna amministrazione) disciplinati da provvedimenti che, tutti, rinvengono la loro stella polare nel dovere di fedeltà alla Repubblica e nell'art. 54 (secondo comma) della Carta costituzionale per il quale «I cittadini cui sono affidate funzioni pubbliche hanno il dovere di adempierle con disciplina ed onore, prestando giuramento nei casi stabiliti dalla legge». Tali codici si inseriscono in un prisma articolato di misure (sostanzialmente) anticorruttive di diverso genere adottate da una sequenza cronologicamente protratta di interventi normativi volti ad una maggiore efficacia, con l'«intenzione $[. .$.$] di recuperare la credibilità delle amministrazioni, la cui integrità non$ può che essere correlata alla persona del dipendente che rappresenta l'ente dinnanzi agli amministrati» ${ }^{24}$, nonché di fomentare lo spirito di corpo che deve raccordare pubblici dipendenti e fruitori dei servizi. Lo scopo è quello di com-

${ }^{21}$ E. BAURA, Il "buon governo"..., cit., 23.

${ }^{22}$ E. BAURA, $I l$ "buon governo"..., cit., 26.

23 Ad esempio l'Organizzazione per la Sicurezza e la Cooperazione in Europa (OCSE) da tempo sollecita presso gli Stati l'adozione di codici etici per il buon governo e per promuovere l'integrità del servizio pubblico.

24 R. CARIDÀ, Codice di comportamento dei dipendenti pubblici e principi costituzionali, federalismi.it. Rivista di diritto pubblico italiano, comparato, europeo 14 (2016) n. 25 9. Cfr. anche E. CarLONI, Il nuovo codice di comportamento e il rafforzamento dell'imparzialità dei funzionari pubblici, Istituzioni del federalismo 33 (2013) 377 ss. 
battere con armi sempre più valide quei fenomeni che la dottrina anche italiana ha sintetizzato nell'anglicismo maladministration: secondo la definizione di Sabino Cassese, giudice emerito della Corte costituzionale, «"Desgobierno", "administrative malpractice", "maladministration": questi i termini con i quali viene indicato quel complesso di disfunzioni che va dalle resistenze al cambiamento al formalismo, all'indifferenza all'efficienza, all'ostilità verso la tecnologia, all'"overstaffing", al nepotismo, alla corruzione ${ }^{25}$. E le armi sono non solo, o primariamente, quelle penali, riservate alle fattispecie appunto penalmente rilevanti. Anzi, nelle ipotesi di altro tipo e che comunque generano situazioni di illegittimità ${ }^{26}$, le armi, amministrativistiche, della responsabilità disciplinare ${ }^{27}$ hanno una più vasta area di esplicazione e si dimostrano alquanto penetranti: soprattutto sul piano della prevenzione, come si è notato, anche per scalfire un pervasivo malcostume culturale, sì che si rinsaldino comportamenti non solo rispettosi delle norme ma deferenti dell'etica pubblica, non rinchiudendosi nella mera sinallagmaticità delle obbligazioni corrispettive a contenuto patrimoniale; senza contare, per altro versante, come ciò si riversi sul miglioramento delle performances delle amministrazioni pubbliche medesime. Occorre un impegno prolungato e su differenti fronti, non abbassando mai la guardia, ma, gradino per gradino, la vetta va raggiunta. E 'gradini', come proprio quello della messa in opera di codici di comportamento, pubblici e sottoscritti dal dipendente all'atto dell'assunzione, si sono dimostrati assai virtuosi: i doveri connessi allo status di dipendente pubblico «trovano nelle fonti normative primarie una specificazione e nei codici di comportamento una esemplificazione ulteriore della condotta pretesa ${ }^{28}$ dalla Costituzione nei confronti delle amministrazioni (datore di lavoro) e degli amministrati (al servizio dei quali si pone, per definizione, l'attività pubblica)» ${ }^{29}$; il codice di comportamento «si configura come interpretazione che in un dato momento storico si

25 S. CASSESE, «Maladministration» e rimedi, Il foro italiano 127 (1992) V 244.

${ }^{26}$ Cfr. le precisazioni di M. Clarich - B. G. MatTARElLa, La prevenzione della corruzione, in B. G. Mattarella - M. Pelissero (eds.), La legge anticorruzione. Prevenzione e repressione della corruzione, G. Giappichelli Editore, Torino 2013, 61.

27 Cfr., per qualche indicazione, con riferimenti giurisprudenziali, il contributo recente di P. MATTEINI - L. ORSINI, Il procedimento disciplinare dei dipendenti pubblici fra modifiche legislative e giurisprudenza della Corte di Cassazione 2015/2016 - Aran, Occasional paper 6/2016, http://www.arangenzia.it/index.php/statistiche-e-pubblicazioni/.

28 Ancora attuale sui doveri del personale la ricostruzione di A. M. SANDULLI, Manuale di diritto amministrativo, Jovene, Napoli 1980, 213 ss.

29 R. CARIDÀ, Codice di comportamento..., cit., 7. 
offre dei doveri costituzionali la cui funzione è sicuramente esemplificativa e consente ai lavoratori pubblici di conoscere, anche se in termini generali, quali sono i comportamenti sicuramente non ammessi, non solo verso il datore di lavoro pubblico, ma pure verso la collettività degli amministrati ${ }^{30}$.

Certo non è questa la sede per una disamina analitica del modello italiano dei codici di comportamento né, tanto meno, delle sue applicazioni: e non perché non sarebbe proficuo inoltrarsi sulle soluzioni realizzate (oltre che per flettere e quasi sillabare i principi costituzionali in condotte concrete dei dipendenti - operazione utilissima poi anche in eventuale sede giudiziale -, pure anche, ad esempio, per recepire proposte, istituire 'punti di ascolto' o raccogliere le segnalazioni 'dall'esterno'), ma unicamente perché eccederebbe l'economia di questo lavoro. Ne abbiamo rapidamente trattato solo per suggerire una pista di ricerca che non ci sembrerebbe così stravagante anche in Ecclesia, naturalmente sempre avendo riguardo alla specificità della sua realtà, oltre che alla peculiarità nonché eterogeneità dei vari officia et munera. Quello che in particolare preme segnalare è come alla base della 'strategia' di queste esperienze normative si collochi sempre il perseguimento della trasparenza completa e della specchiata imparzialità ed equità nell'erogazione dell'attività amministrativa, della formazione (periodicamente iterata), della selezione e del rendimento delle risorse umane, della documentabilità dei processi decisionali (per le verifiche), e soprattutto l'incentivo alla sensibilizzazione dei «cittadini utenti, nel rispetto della loro dignità» ${ }^{31}$ : essi hanno il diritto di agire (per far valere i relativi obblighi) e di intervenire con denunce ed esposti esercitando i diritti di partecipazione a difesa della loro pretesa a «un comportamento improntato a disciplina e onore in coloro cui sono state affidate pubbliche funzioni» ${ }^{32}$ (tra l'altro sempre più avvertita è l'esigenza anche di tutelare le persone che denunciano abusi nel settore pubblico).

Se dunque gli ordinamenti secolari perseguono il buon governo attraverso la scelta e il controllo di persone preparate e rette, l'ordinamento cano-

${ }^{30}$ G. M. RACCA, Disciplina e onore nell'attuazione costituzionale dei codici di comportamento, in F. MERLONI - R. Cavallo Perin (eds.), Al servizio della Nazione. Etica e statuto dei funzionari pubblici, Codice Edizioni, Milano 2009, 260. Cfr. anche B. G. MATTARELla, Le regole dell'onestà. Etica, politica, amministrazione, il Mulino, Bologna 2007, 139.

${ }^{31}$ V. Cerulli Irelli, Per una politica dell'etica pubblica nella disciplina delle funzioni amministrative, Astrid 14/2019, www.astrid-online.it 2.

32 R. CAVAllo PERIN, L'etica pubblica come contenuto di un diritto degli amministrati alla correttezza dei funzionari, in F. MERLONI - R. Cavallo Perin (eds.), Al servizio della Nazione..., cit., 161. 
nico, in virtù del suo superiore approccio sapienziale cristiano, non dovrebbe orgogliosamente e superbamente - nonché miopemente - disdegnare certi spunti evincibili da regole e procedure statali: certo senza velleitarie idealizzazioni, senza appiattimenti insensati e senza mai avvilire l'umanità e la personalità dei rapporti. Invero una sorta di codice di comportamento è racchiuso, ad esempio, nello stesso Regolamento generale della Curia romana del 30 aprile 1999, segnatamente nel Titolo VI, Doveri del personale - mentre il Titolo X è dedicato alle Norme disciplinari (sanzioni e procedura) ${ }^{33}$-, nel quale «Alcuni dei doveri elencati, oltre a derivare da normali esigenze di professionalità applicabili a qualsiasi rapporto lavorativo, possono essere considerati "norme di salvaguardia dell'identità" poiché tengono conto della specificità del lavoro della curia, così come del contegno cristiano e della naturale riservatezza dovute al genere di funzioni che si esercitano» ${ }^{34}$. Ed è vero altresì, si potrebbe ribattere, che un codice comportamentale prioritariamente 'cristiano' è ricostruibile anche da un mosaico di norme (che pure impartiscono sanzioni disciplinari) da cui anche le codificazioni canoniche sono punteggiate: ma non solo.

Certamente non intendiamo addentrarci ora nella schermaglia sulla 'sostanza' e sulla 'consistenza' del diritto disciplinare canonico, sulle sue connessioni/distinzioni con il diritto penale e con il diritto amministrativo, sulla sua necessaria/opportuna 'codificazione' - universale o particolare - con le relative sanzioni e le procedure per comminarle (anche qui in rapporto con quelle penali e amministrative). Si tratta di un tema assai interessante e molto vasto, non troppo esplorato, ove tra l'altro non di rado gli illeciti o infrazioni disciplinari - violazioni di doveri dell'ufficio (sempre in senso latissimo), atti contrari allo status assunto con un impegno - possono incrociarsi con la mancanza di idoneità dei titolari degli uffici ovvero con la perdita dei requisiti per l'accesso ai medesimi, con una 'liquidità' di confini tra gli ambiti: ma si tratta di un tema che chiaramente esorbita quel 'diritto alla denuncia' di cui ci stiamo occupando. In questa sede ci limitiamo ad osservare che proprio l'esperienza degli ordinamenti secolari - anch'essi assisi su principi costituzionali sovraordinati, sia pur di diversa 'consistenza' - ha comprovato che talora una metodica stesura di regole e procedure per ogni singola 'amministrazione',

${ }_{33}$ Cfr. Regolamento generale della Curia romana, 30 aprile 1999, AAS 91 (1999) 629-699.

${ }^{34}$ J. I. ARRIETA, Funzione pubblica e attività di governo nell'organizzazione centrale della Chiesa: il Regolamento generale della curia romana, Ius Ecclesiae 4 (1992) 595. 
adattate alla medesima e attente ai problemi via via sorgenti, è stata tutt'altro che inane: a maggior ragione i disposti indirizzati a chi opera nella Curia romana, nei quali il valore parenetico si amalgama con il dovere morale e giuridico, non dovrebbero restare inimitati 'extra Urbem' ${ }^{35}$. E come ai cittadini in queste regole e procedure è consegnato un ruolo di impulso per sollecitare alla vigilanza le autorità incaricate - tanto che la dottrina giunge ad enucleare ed appoggiare l'avvio di forme di partecipazione degli 'amministrati' assai 'invasive' che scuotano una spesso immobile e monolitica autoreferenzialità del potere pubblico e impongano la riparazione degli errori commessi - così nella Chiesa è intuibile che possano essere soprattutto i fedeli, i quali entrano in contatto con i titolari di uffici, ad affacciarsi in primo piano con la loro 'denuncia' per evitare pregiudizi futuri: riempiendo di contenuto - e in questo ogni raffronto con i doveri civici impallidisce - la loro vera aequalitas quoad dignitatem et actionem (can. 208) col coadiuvare la buona amministrazione della Chiesa, secondo d'altronde una pratica inveterata dell'epopea cristiana seppur in cangianti sembianze ${ }^{36}$.

\section{DAL DIRITTO Di PETIZIONE DEI FEDELI (CAN. $212 \$ 2$ ) ALLA RIVENDICAZIONE DI UN INTERESSE DIFFUSO}

Ma giungendo al cuore del nostro ragionamento volto a costruire una sorta di abilitazione giuridica della comunità a reagire dinanzi alla latitanza, all'inerzia e all'attendismo dell'autorità preposta alla designazione e alla supervisione sui nominati, esplicando il 'versante doveroso' del diritto al buon governo, possiamo tracciare le ulteriori tappe di una plausibile rotta, senza tacerne le difficoltà giuridiche.

Anzitutto può essere sicuramente addotto il 'diritto di petizione' riconducibile al can. $212 \$ 2$ e a tutti ben noto, esonerandoci qui da un’illustrazione dettagliata. Avanzare una petizione - ben motivata e circostanziata, basata su un fermo convincimento - «non è semplicemente una possibilità lasciata all'arbitrio della gerarchia» ${ }^{37}$ : rispetto inoltre al già considerato para-

${ }^{35}$ Cfr. quanto osserva M. Rivella, Il diritto disciplinare della Curia Romana, Quaderni di diritto ecclesiale 31 (2018) 320.

${ }^{36}$ Cfr. recentemente i rilievi di J. CANOSA, La rilevanza della collaborazione attiva dei fedeli per la buona amministrazione ecclesiastica, Ius Ecclesiae 30 (2018) 17 ss.

37 J. Hervada, Diritto costituzionale canonico, prefazione all'edizione italiana di G. Lo Castro, Giuffrè Editore, Milano 1989, 137. 
grafo terzo, «l'opinione non sempre richiede una risposta, mentre al diritto di petizione corrisponde necessariamente il dovere di rispondere ${ }^{38}$. Sul punto personalmente non possiamo che sottoscrivere i generosi conati della canonistica più evoluta che preme sia per corroborare la cogenza della risposta dei superiori - ovviamente nei limiti della ragionevolezza, senza oberare sconsideratamente questi ultimi -, sia per dilatare quanto più la presunzione di risposta negativa (il silenzio rigetto $e x$ can. 57) al fine di ampliare, laddove sussistano i presupposti, l'esperibilità di uno strumento giuridico di tutela: cioè per rendere esigibile un solerte e giusto riscontro motivato almeno succintamente (quando non affermativo), non certo per coartare un automatico accoglimento della petizione. Essa potrebbe profilarsi proprio come interpellanza, esposto o reclamo al fine di ottenere il ripensamento e la rivisitazione di una nomina, cioè in funzione dell'adozione di un provvedimento correttivo di governo: il suo oggetto avrebbe un respiro compiutamente giuridico, ancorandosi sul diritto del fedele ad un esercizio corretto della funzione gerarchica, anche quando non lo tange in maniera immediata. Tra l'altro il diritto di rendere noti all'autorità ecclesiastica, centrale e locale, certi dati ad essa rimasti ignoti e che invece sono suscettibili di rivestire un ruolo non collaterale potrebbe configurarsi quale dovere per il fedele, almeno morale. Questi poi, oltre a ragguagli circa il difetto delle postulate qualità nel nominato, potrebbe, come anticipato, appuntare le sue querimonie su vizi e pecche proprio nel procedimento - laddove in qualche modo normato, anche se non a livello legislativo: da qui l'importanza di perfezionare quella razionalizzazione e formalizzazione cui abbiamo sopra alluso - di designazione, sì che l'idoneità non è stata adeguatamente appurata (o addirittura si è minimizzata o occultata l'inidoneità), omettendo di comprovarla in maniera soddisfacente; oppure ancora denunciare come si sia simulato di assumere le dovute conoscenze, che pure il Codice prescrive di conseguire, solo per legittimare una scelta già preconfezionata e parziale. Tra i fedeli, inoltre, potrebbero forse più degli altri motivatamente presentare tale petizione, per lo meno di chiarimento, coloro che sono stati consultati nell''istruttoria' e hanno dato parere negativo (can. 50) disatteso poi dall'autorità (che deve motivare, saltem summarie, ex can. 51), sine praevalenti ratione: si consideri infatti a riprova quanto sancisce il can. $127 \$ 2$.

${ }^{38}$ E. BAURA, $I l$ "buon governo"..., cit., 21. 
Sinora abbiamo discorso principalmente di eventuali interventi dei fedeli - in scienza e coscienza - che si sostanziano però unicamente, anche confidenzialmente ed ufficiosamente, in pareri, sententiae, osservazioni, moniti, petitiones: in una 'denuncia', cioè, genericamente intesa. Ma la linea di demarcazione rispetto a un vero e proprio atto di iniziativa ci pare che possa, segnatamente in reazione all'ignavia o alla stasi dell'autorità ecclesiastica, pure avvisata, farsi sempre più gracile: fino a rendere quasi 'spontaneo' il trapasso dalla mera 'denuncia' del tutto inascoltata ad un atto appunto meno informale e in grado di mettere finalmente e più cogentemente in moto l'autorità affinché si provveda. Viene in rilievo la cosiddetta remonstratio o richiesta previa, denominata anche supplicatio ovvero proprio petitio sì che gli istituti tendono a confondersi, anche se può tratteggiarsi un discrimine, sembrando insita nella remonstratio proprio la contrariata 'rimostranza', cioè una contestazione fondata su fatti provati o provabili: con essa si intende, come risaputo, la petizione previa al ricorso gerarchico all'autore del decreto chiedendo per iscritto la sua revoca o correzione secondo il dettato di cui al can. 1734 $\$ 1$. Uno snodo forse sottovalutato e che vale invece la pena 'rilanciare' in qualche modo come chance risolutoria precoce ed anticipata da non sprecare: rappresenta infatti la 'circostanza' in cui l'autorità viene frontalmente messa dinanzi alle sue responsabilità e all'eventuale inadeguatezza del suo atto, potendo essere indotta alla revisione dello stesso al cospetto dei dati ed elementi veicolati esaurientemente dalla remonstratio medesima - da quella ob illegitimitatem più che da quella ob gravamen -, e così risparmiando 'prosecuzioni contenziose' sicuramente più defatiganti e laboriose ${ }^{39}$, oltre che incresciosamente 'litigiose'.

Tuttavia nella remonstratio il fedele solitamente è il diretto destinatario dell'atto che lo concerne personalmente: mentre la prospettiva qui investita è quella che non sia - si intuisce - colui cui è stato devoluto l'incarico a innescare l'emenda del provvedimento, o colui che è stato 'scartato' per la nomina, oppure ancora chi è stato menomato nelle sue spettanze a cagione dell'inidoneità del designato, ma l'iniziativa provenga del tutto ab extrinseco da portatori di utilità ecclesiali non direttamente proprie, anzi con ricadute comunitarie. Del pari, facendo però un passo indietro e riprendendo ancora una volta in mano

39 Cfr. P. GHERRI, L'autotutela amministrativa come supplemento di conoscenza: la remonstratio canonica (Cann. 1732-1734 CIC), cit., 357 ss., 375 ss.; IDEM, Petitio, remonstratio, exceptio. Cenni esplorativi sui modi di non-esecuzione degli atti amministrativi singolari, Ius Ecclesiae 27 (2015) 350 ss. 
il canone $212 \S 2$, esso parla invero di necessitates suas e optata sua; al di là di ogni concessione ad un soggettivismo individualista, sempre un fraintendimento in Ecclesia, pure la petizione sembrerebbe implicare l'appagamento di propri interessi, sui quali la duplicazione del possessivo parrebbe calcare. E se si è patrocinato che «un sistema più attento alla tutela effettiva dei diritti dei fedeli dovrebbe garantire in maniera speciale il diritto di petizione e riconoscere con ampiezza la correlativa legittimazione attiva per ricorrere contro la sua violazione» ${ }^{40}$, ciò si rivela più ostico quando si vuol far valere un interesse in qualche modo sopraindividuale.

Ecco quindi che sovviene immediatamente il dibattito sulla configurabilità degli interessi collettivi o, meglio, di quelli diffusi nello ius canonicum, cioè quelli «comuni ad una pluralità di soggetti, non identificati in base alla preesistenza di rapporti giuridici rispetto al bene e riferibili non al soggetto come individuo, ma come membro di una collettività più o meno ampia, coincidente, al limite, con la generalità dei cittadini» ${ }^{41}$. Un dibattito qualche anno addietro assai acceso, forse in qualche misura anche trascinato dalla frustrazione ancora cocente delle attese in merito alla giustizia amministrativa (specie 'decentrata') nella Chiesa, in un 'cantiere' che si intendeva mantenere aperto. Ma se la querelle appare oggi meno vivace, tuttavia i tentativi di allargare le maglie della legittimazione al ricorso amministrativo, perseguiti con attenuato clamore ma non dimidiata tenacia, hanno attinto, tra l'altro, giurisprudenzialmente risultati non trascurabili, forse propiziati da un'atmosfera maggiormente distesa. Ci basti ora rimarcare come le disquisizioni dei canonisti presentino sovente il minimo comune denominatore di puntare a sfruttare al meglio le pieghe dell'ordinamento per la rivendicabilità in senso lato di tali interessi metaindividuali o adespoti, come vengono etichettati: cercando in particolare di imprimere una lettura elastica al - pur ferreo (ma non immune da discrepanze sviscerate in dottrina) - famoso decreto della Segnatura del $1987^{42}$, per il quale l'interesse adducibile avrebbe dovuto comunque essere

${ }^{40}$ E. BAURA, Analisi del sistema canonico di giustizia amministrativa, in E. BAURA - J. CANOSA (eds.), La giustizia nell'attività amministrativa della Chiesa: il contenzioso amministrativo, Giuffrè Editore, Milano 2006, 20.

${ }^{41}$ P. MONETA, I soggetti nel giudizio amministrativo ecclesiastico, in La giustizia amministrativa nella Chiesa, Libreria Editrice Vaticana, Città del Vaticano 1991, 62.

42 Cfr. il Decreto 21 novembre 1987 del Supremo Tribunale della Segnatura Apostolica, Castillo Lara ponente, Communicationes 20 (1988) 92-94. Cfr. anche il Responso del 20 giugno dello stesso anno della Pontificia Commissione per l'interpretazione autentica del Codice di Diritto Canonico, AAS 80 (1988) 1818. 
«personale, directum, actuale et a lege, saltem indirecte, tutelatum», e che ha rischiato di ostruire ogni prospettiva. Prospettive che pure il Codex Iuris Canonici post-conciliare aveva in qualche modo dischiuso con l'assai ampia dizione del can. $1737 \$ 1$ quanto invero al ricorso gerarchico (ma le aspettative non possono essere rigidamente scisse): «propter quodlibet iustum motivum». Per aggirare lo scoglio gli sforzi si sono addensati soprattutto sulla personalità dell'interesse, per renderlo più duttile e malleabile, non sinonimico e tale da non ridursi all'esclusività: «nell'ordinamento della Chiesa il termine personalità non indica una titolarità esclusiva, ma una titolarità "indipendente" del singolo. Titolarità indipendente intesa come spettanza di una certa posizione giuridica in capo a un singolo soggetto il quale ha facoltà di azionarla liberamente (cf dunque la personalità dell'interesse), ma che non può accampare la pretesa di essere l'esclusivo titolare di tale situazione e del bene tutelato a essa sotteso (cf dunque la mancanza di esclusività dell'interesse)» ${ }^{43}$. E, come anticipato, piccoli (e anche meno piccoli) steps nella giurisprudenza, sotto le pressioni pressoché unisone della dottrina, si sono compiuti, con gradualità ma con pervicacia - sia pur, va convenuto, con qualche inciampo e asperità ${ }^{4+}$ - per una sempre maggiore giustiziabilità degli interessi plurisoggettivi: ciò che non può non effondersi ordinamentalmente, per così dire.

Vale forse dunque la pena incedere oltre su questo tragitto, spezzando una lancia perché si compia un passaggio ulteriore che miri ad allargare ancor più lo spettro di quel 'personale', slegandolo da indici di personalizzazione troppo tetragoni e anchilosati e da più o meno larvate fictiones di personalità o para-personalità. E lo diciamo qui senza avere principale riguardo all'ambito della normativa sui ricorsi veri e propri, ma con il grandangolo rivolto ad una più generica e dilatata legittimazione all'iniziativa e attività concreta da parte dei christifideles: legittimazione avvinta tra l'altro ad una concezione di diritto nella Chiesa finalmente emancipata da ogni visione individualista di stampo liberale o comunque secolare. $\grave{E}$ forse proprio in quel momento liminale, in quella «prima istanza "sostanziale" ${ }^{45}$ che occorre

${ }^{43}$ G. TOGNONI, La tutela degli interessi diffusi nell'ordinamento canonico, Quaderni di diritto ecclesiale 8 (1995) 332-333.

${ }^{44}$ Cfr. quanto già riferiva sull'evoluzione della giurisprudenza I. ZuANAZZI, La legittimazione a ricorrere uti fidelis per la tutela di diritti comunitari, in R. BERTOLINO - S. GHERRO - G. LO CASTRO (eds.), Diritto 'per valori' e ordinamento costituzionale della Chiesa, G. Giappichelli Editore, Torino 1996, 427 ss.

45 P. GHerri, Petitio..., cit., 344. 
aguzzare l'attenzione, bandolo di ogni successivo stadio giuridico (e eventualmente anche per risolvere anticipatamente il problema senza 'complicanze' contenziose). Oggi con sempre maggiore nerbo si rivendica: «la guida, la presidenza o la rappresentanza di una comunità, con gli indubbi obblighi qualificati di cura e custodia che ne derivano, non implicano però la singolarità ed esclusività delle attribuzioni e, soprattutto, l'estromissione della titolarità della collettività. Fermo restando il fatto che l'organizzazione ecclesiastica non è meramente pratica o funzionale ma configura $\mathrm{i}$ beni e $\mathrm{i}$ vincoli della comunione, interessa evidenziare che l'azione pubblica è una spettanza dell'intera comunità dei discepoli di Cristo. La stessa gerarchia integra e "colora" il bene comune ecclesiale. Condividere significa allora aderire o prendere parte alle scelte e decisioni che riguardano la vita della comunità. Non si tratta invero di un'inconcepibile istanza populistica e democratizzante, ma di una necessaria forma di scambio e dialogo tra autorità e amministrati. I fedeli non sono essenzialmente sudditi o sottoposti ma destinatari e beneficiari della cura pastorale e perciò partecipi e consorti del retto ordine sociale» ${ }^{46}$. 'Denunciando' l'inidoneità di taluno ci si mette in moto, pertanto, non per fruire 'privatamente' di un'utilità o godere di un bene, né per accrescere il proprio appannaggio o la propria sfera giuridica, ma per affinare la qualità dell'amministrazione ecclesiastica: senza che ciò debba più venire patito come intralcio o ingerenza da una visione elitaria, aristocratica e al fondo gerarcologica del potere. Tornando alla pericope della Segnatura, che poi il diritto al buon governo sia «tutelatum a lege salte indirecte» non ci pare possa essere infirmato, attesa anche la portata, non certo riduttivamente giuspositiva, da devolvere a 'legge canonica' in casu. Mentre l'indice di soggettivazione' che sospingerebbe il fedele ad agire e simultaneamente ne coonesterebbe la legittimazione propria, nonostante la teorica riproduzione seriale in altri co-latori, anzi nell'intera comunità, oltre che nella localizzazione dell'ufficio de quo nella comunità di cui il christifidelis si sente partecipe attivo e 'militante', e dunque nelle interrelazioni col medesimo in virtù dell'incorporazione (alla diocesi, per esempio), ci sembra si possa radicare proprio nella ben formata sententia del fedele (cfr. il can. 212, nell'interconnessione tra paragrafo 2 e paragrafo 3 ) che diviene sicura conoscenza dell'inidoneità all'ufficio di colui al quale è stato assegnato.

${ }^{46}$ M. Del Pozzo, Lo statuto giuridico..., cit., 141. 


\section{DALl'obbligo Di RIVELARE IMPEDIMENTI AL MATRIMONIO E AI SACRI ORDINI...}

Va posto al proposito in risalto che non sono rari i casi rinvenibili, e non da oggi, nell'ordinamento canonico in cui i christifideles sono convocati a dichiarare quanto è a loro conoscenza al fine di evitare detrimento al bonum publicum. $\mathrm{Al}$ di là della vera e propria denuncia penale di delitti che si pone su un distinto crinale e che ora rischierebbe di condurci fuori strada, un caso assai eclatante e che ci pare appropinquarsi ai ragionamenti sviluppati afferisce all'ambito delle pubblicazioni che devono precedere lo scambio dei consensi nuziali. Da tempo risalente nella Chiesa ci si è industriati per scongiurare la contrazione di vincoli che si dimostrassero poi viziati; e dopo la piena acquisizione dell'inclusione del matrimonio tra battezzati nel settenario sacramentale, anche i banni nuptiales sono convogliati a preservare la validità di un sacramento. A quest'opera di custodia è vocato a cooperare l'intero popolo di Dio - «inquirere una cum populo ipse sacerdos debet ${ }^{47}$ ammonivano antiche fonti altomedievali - che diviene garante del retto esercizio dello ius connubii: una convocazione dal possente significato evocativo, tra l'altro già lanciato in un tempo in cui ancora si doveva 'riscoprire' in tutta la sua pregnanza la corresponsabilità dei cbristifideles in una societas Ecclesiae in procinto di essere egemonizzata dal 'duo genera cbristianorum'. E, come di consueto, all'informazione (publicatio) deve seguire l'azione. Così ancora il vigente can. 1069 fissa in capo ad ogni fedele senza alcuna distinzione, prima della celebrazione del matrimonio, l'obbligo di rivelare al parroco o all'ordinario del luogo gli impedimenti di cui sia a conoscenza in relazione al matrimonio del quale si è dato annuncio erga omnes: impedimenti che vanno latamente intesi come obices irritanti anche di altro genere rispetto agli impedimenta propriamente detti. È palese qui come il christifidelis non possa vantare nessun interesse 'personale' nell'assolvere a questo diritto-obbligo di allertare l'autorità ecclesiastica sì che si possano assumere provvedimenti.

Pur essendo il matrimonio una relazione tra i due coniugi, esso eccede il loro privato interesse e ciò giustifica una compressione del diritto alla propria intimità (can. 220), motivata da un atto di carità nei loro confronti, evitando una situazione virtualmente enutritiva peccati. $\grave{E}$ innegabilmente la rilevanza 'sociale' e di bene pubblico del coniugio - sul quale germina la famiglia, cellula vitale della società civile non meno che della comunità ecclesiale -, nonché

${ }^{47}$ La citazione si riferisce a Benedetto Levita che riproduceva un capitolare carolingio dell' 802. 
la sua 'elevazione' a segno efficace della grazia divina se contratto tra battezzati, e quindi il diritto divino che comanda di non permettere l'irriverenza a un sacramento, a radicare l'obbligazione (in sé di diritto ecclesiastico) gravante sui fedeli (non solo peraltro). E se questa è acquisizione pluricentenaria, ci pare oggi viepiù da rinfocolare tale mentalità di compartecipazione dei fedeli alla 'salute' di un matrimonio, da cui trapela un risvolto diremmo istruttivo di densità pastorale di grande pregio in un'epoca scristianizzata e di imperante "individualismo libertario" 48: ancora nel 1989 la Conferenza Episcopale Italiana si rincresceva che, benché il diritto canonico contemplasse alcune forme di partecipazione aperte a tutti i cristiani, esse, fra cui proprio «dare le informazioni richieste in ordine all'ammissione dei candidati alla celebrazione dei sacramenti che hanno speciale rilievo "sociale" (pubblicazioni matrimoniali, informazioni relative ai candidati al sacerdozio)», «spesso sono disattese o nella loro pratica o nell'impegno a viverne l'originario significato» ${ }^{49}$.

Sempre in questo contesto va pure ricordato che l'«impugnazione della validità del matrimonio è stata per lungo tempo consentita a qualunque cattolico» ${ }^{50}$. Nello ius decretalium la legittimazione all'accusatio era estesa anche a persone estranee al rapporto sponsale, pure talora a quilibet de populo, e la denuntiatio poteva essere presentata da chiunque. Invero poi il can. $1971 \mathrm{del} \mathrm{Co-}$ dex Iuris Canonici del 1917 «suprimió la acusación popular» ${ }^{51}$ e sancì che erano abili ad accusare il matrimonio i coniugi $(\$ 1 \mathrm{n}$. 1: «in omnibus causis separationis et nullitatis, nisi ipsi fuerint impedimenti causa») e il promotore di giustizia ( $\$ 1$ n. 2: «in impedimentis natura sua publicis»), mentre «Relinqui omnes, etsi consanguinei, non habent ius matrimonia accusandi, sed tantummodo nullitatem matrimonii Ordinario vel promotori iustitiae denuntiandi» (\$2). E oggi il canone 1674, anche nella versione esito del Motu Proprio Mitis iudex Dominus Iesus del 15 agosto $2015^{52}$, recita al primo comma 'Sono

48 L'espressione è di papa FranCESCO, Messaggio alla Professoressa Margaret Archer, Presidente della Pontificia Accademia delle Scienze Sociali in occasione della sessione plenaria, 24 aprile 2017, consultabile online all'indirizzo www.vatican.va.

49 Conferenza Episcopale Italiana, Comunione, comunità e disciplina ecclesiale. Documento dell'episcopato italiano, $1^{\circ}$ gennaio 1989, n. 75 .

50 P. MONETA, I soggetti nel giudizio..., cit., 61.

51 R. Rodríguez-Ocaña, Sub can. 1674, in Á. MarzoA - J. Miras - R. RodrígueZ-Ocaña (eds.), Comentario exegético al Código de Derecho Canónico, IV/2, tercera edición actualizada, Eunsa, Pamplona 2002, 1851.

52 Cfr. Francesco, Litterae Apostolicae Motu Proprio datae Mitis iudex Dominus Iesus, AAS 107 (2015) 946-957. 
abili ad impugnare il matrimonio: $1^{\circ}$ i coniugi; $2^{\circ}$ il promotore di giustizia, quando la nullità sia già stata divulgata, se non si possa convalidare il matrimonio o non sia opportuno': non viene pertanto più esplicitamente codificato l'istituto della denuncia. Non è questa la sede per approfondire i motivi di tale mutamento disciplinare - specie per quanto concerne i coniugi - scandito nell'arco del tempo da un susseguirsi, anzi da uno stillicidio di interventi di rifinitura. Senza tema di smentite può però affermarsi che esso, sul punto specifico, non è certo avvenuto per esautorare il populus Dei affinché non si frammettesse e immischiasse nelle faccende altrui, ma primariamente allo scopo di non travolgere valori umani e personali insiti in situazioni, pur eventualmente antigiuridiche, che si sono però stabilizzate, e di rispettare la libertà e l'autodeterminazione del singolo in ordine all'accertamento del proprio status. Perciò non a chiunque ma unicamente - oltre ai coniugi senza limitazioni, ma non ad altre persone - e in qualche modo straordinariamente alla 'parte pubblica', venuta a conoscenza di un qualche caput nullitatis, è rimessa la decisione di sottoporre il matrimonio a processo per pervenire alla dichiarazione dell'invalidità: e appunto «Soltanto quando la nullità diventa di dominio pubblico, provocando turbamento e sconcerto (o scandalo, come un tempo si usava dire) $\gg^{53}$ e non sia possibile la convalidatio. Come è stato sunteggiato, «La forte limitazione della capacità di essere parte attrice (ristretta praticamente ai soli coniugi: cfr. DC art. 92 n. 1), introdotta dal CIC 1917 esprime il desiderio di proteggere la stabilità del matrimonio (il favor matrimonii) e tutela l'intimità coniugale. Fino alla prima codificazione era vigente il diritto delle Decretali (cfr. X.4.18), in forza del quale (per molti secoli) la capacità di essere parte attrice era riconosciuta anche ad altre persone, in particolare ai familiari di entrambi i coniugi, poiché si riteneva che vivere come coniuge senza esserlo - per la nullità del matrimonio - significava vivere da peccatore pubblico, sicché questa ratio peccati giustificava un'ampia legittimazione per impugnare il matrimonio. La legittimazione sussidiaria del promotore di giustizia per impugnare la validità del vincolo [...] mantiene un fondamento analogo e, frequentemente, presupporrà la denuncia di coloro che, secondo il diritto precodiciale, avrebbero potuto impugnare la validità del matrimonio ${ }^{54}$. Con la denuncia i fedeli farebbero valere un'“aspettativa diffusa" esplicativa di un

${ }^{53}$ P. MonetA, Introduzione al diritto canonico, quarta edizione, G. Giappichelli Editore, Torino 2016, 50-51.

${ }^{54}$ J. LLOBELl, I processi matrimoniali nella Chiesa, Edusc, Roma 2015, 179-180. 
"tipico interesse ecclesiale" ${ }_{55}$ relativo alla validità o no del matrimonio. Invece, come appena constatato, è ancor oggi rimasta la previsione espressa del - forse meno 'traumatico' e più 'fisiologico' - impegno preventivo accollato ad ogni christifidelis, sempre orientato al bonum commune, di evitare che tali situazioni antigiuridiche, se vogliamo così continuare a definirle, vengano ad esistenza, 'denunciando' ciò che sanno all'autorità ecclesiastica.

Forse ancora più indicativo in relazione al tema sondato in queste pagine il diritto-dovere dei fedeli di rivelare, se ne sono a conoscenza, gli impedimenti ai sacri ordini all'ordinario o al parroco ante ordinationem secondo quanto intima il can. 1043: a questo dovere si correla la prassi, pur non prevista come obbligatoria dal Codex Iuris Canonici - anche in passato nella Chiesa ci si rimetteva solitamente agli statuti diocesani e alle usanze locali -, delle pubblicazioni canoniche, richieste peraltro da una Lettera Circolare della Congregazione per il culto divino e la disciplina dei sacramenti del $1997^{56}$. Va puntualizzato che il lemma 'impedimento' deve essere anche qui inteso in senso ampio, inclusivo parimenti delle irregolarità, ed inoltre, se è vero che il precetto «solo obliga a manifestar los impedimentos para la recepción de órdenes, pero no la ausencia de otros requisitos necesarios para la licitud de la ordenación, como no haber recibido el sacramento de la confirmación. No obstante, la responsabilidad de los fieles en orden a la idoneidad de los candidados al sacramento del orden no se agota en el cumplimineto de este canon» ${ }^{57}$. D'altronde è irrefragabile che l'idoneità è intimamente avviluppata con irregolarità e impedimenti: «el tema de la idoneidad de un candidato al orden también está presente, de una forma u otra, en los cánones que se occupan de las irregularidades y los impedimentos para la ordenación» ${ }^{58}$. Come si è postillato, «La normativa evidenzia con questo canone un dovere dei fedeli relativo a contribuire all'iter dell'accesso agli ordini sacri, un dovere riguardante il tema del discernimento e della valutazione dell'idoneità dei candidati

55 Così M. Miele, Ancora sul promotore di giustizia, JusOnline 2/2018 73 anche in nota 59.

56 Cfr. Lettera Circolare della Congregazione PER IL CUlTo DIVINO E LA DISCIPLINA DEI SACRAMENTI del 10 novembre 1997, Entre las más delicata, Notitiae XXXIII (1997) 495-518, Allegato 2 .

57 J. Ma González del Valle, Sub can. 1043, in Á. MarZOA - J. Miras - R. RodríGueZ-Ocaña (eds.), Comentario exegético al Código de Derecho Canónico, III/1, tercera edición actualizada, Eunsa, Pamplona 2002, 990.

58 A. Miglavacca, Idoneidad para oficios y ministerios, in J. OtaduY - A. VIANA - J. SEDANO (eds.), Diccionario general de derecho canónico, IV, Thomson Reuters Aranzadi, Cizur Menor (Navarra) $2012,364$. 
all'ordine. Questo dovere di partecipazione nasce dalla dimensione di corresponsabilità e di comunione dei fedeli battezzati all'interno della comunità cristiana, che si esercita in particolare anche in vista del discernimento e della promozione dei ministeri ${ }^{59}$. Del resto lo stesso istituto delle irregolarità e impedimenti rinviene la sua ratio nella fissazione di confini proprio alla valutazione discrezionale degli ordinari sull'idoneità per l'ammissione agli ordini sacri in vista dell'oggettiva sussistenza di talune situazioni. Il popolo di Dio, pertanto, adducendo informazioni, arreca un contributo impareggiabile e infungibile in vista dell'attingimento della certezza morale sull'idoneità: un contributo che non si può pretermettere a maggior ragione oggi, in un tempo nel quale si registra che in gran numero giungono al seminario adulti la cui personalità è già definita e con un passato di esperienza di vita sovente non conosciuto o condiviso dai superiori. È incontrovertibile la contiguità di quanto appena detto con il fulcro dei nostri discorsi, che pure non concernono la sola idoneità all'ordine sacro.

\section{6. ...FINO ALLE SUGGESTIONI DISCENDENTI DALL'ANALOGIA CON LE AZIONI POPOLARI: UNA PROPOSTA DE IURE CONDENDO}

Un'ultima provocazione ci pare debba essere lanciata sempre allo scopo di gettare le fondamenta del diritto a 'denunciare' l'inidoneità agli uffici; una provocazione che di primo acchito pare collocarsi in un'orbita totalmente disassata rispetto a quella seguita sinora; e tuttavia non tardano a mostrarsi similitudini, segnatamente con la tematizzazione degli interessi diffusi approcciata sopra, pur con le dovute distinzioni. Una provocazione, ancora, che ci conduce a quell'ambito della iurisdictio Ecclesiae che forse più la distingue e distanzia dagli ordinamenti secolari, marcandone spiccatamente l'irriducibilità: la 'giustizia della santità'.

A parere pressoché unanime, infatti, esisterebbe oggi una sola fattispecie di azione popolare nel diritto canonico, quella che vede quilibet e populo Dei mobilitarsi al fine di promuovere la pronuncia di beatificazione e poi di canonizzazione di un defunto 'in odore di santità': quivis fidelis che chiaramente non vanta alcun interesse prettamente personale alla pronuncia finale né essa tange il suo status. Pur valendo anche per queste cause il principio generale

\footnotetext{
59 A. MigLiavaCCA, Irregolarità e impedimenti: vie di conoscenza e di verifica al servizio del discernimento, Quaderni di diritto ecclesiale 28 (2015) 423.
} 
nemo iudex sine actore, tuttavia «Nessuna limitazione è prevista per la legittimazione ad agire: chiunque faccia parte del popolo di Dio o qualunque gruppo di fedeli può svolgere il ruolo di attore» ${ }^{60}$. Il can. $2003 \$ 1$ del Codex Iuris Canonici del 1917 enunciava: «Quivis fidelis vel legitimus Christifidelium coetus ius habent petendi ut causa apud tribunal competens instruatur», configurando un vero e proprio diritto, sgorgante quale conseguenza del battesimo nella Chiesa cattolica. Nonostante sia stata abolita tale proclamazione espressa, anche nella vigente normativa non è in dubbio l'esistenza di tale diritto, fermo ovviamente che la causa si incardinerà e procederà se ne ricorrono tutti i presupposti. È vero che le Normae servandae in inquisitionibus ab episcopis faciendis in causis sanctorum emanate nel 1983 dalla Congregazione delle cause dei santi ${ }^{61}$ sanciscono che colui che intende promuovere la causa sia 'ammesso dall'autorità ecclesiastica' (art. 1 a): ma si tratta di una semplice «autorizzazione, $[. .$.$] una condizione per l'esercizio di un diritto la cui titolarità$ è riconosciuta dall'ordinamento canonico a tutti i fedeli» ${ }^{62}$ e che è subordinata solo alla verifica che la persona fisica o il coetus abbiano la capacità di assumere gli impegni inerenti al ruolo di attore, addossandosi le responsabilità morali ed economiche della causa. Tra l'altro, sempre le Normae servandae intimano al vescovo di far conoscere pubblicamente la petizione del postulatore nella propria diocesi e, se lo riterrà opportuno, anche nelle altre diocesi, con il consenso dei rispettivi vescovi, invitando tutti i fedeli a dargli notizie utili riguardanti la causa, se ne hanno da fornire (art. 11 b). Questo non solo riporta all'origine 'spontanea' del riconoscimento della santità per moto di venerazione popolare, ma esalta al massimo grado l'apporto del coetus fidelium. La genesi comunitaria e popolare del processo de quo è esplicitata altresì dal requisito, necessariamente da accertare nel processo, della fama sanctitatis (o di martirio, oltre che di 'segni', ovvero anche di 'offerta della vita'): «la presencia activa del Espíritu Sancto en el corazón de los bautizados» ${ }^{63}$, ossia la diffusa persuasione (existimatio seu communis opinio), non artificiosamente procurata, dell'integrità di vita e di un esercizio eccezionale delle virtù cristiane già

${ }^{60}$ P. Moneta, La giustizia nella Chiesa, nuova edizione, il Mulino, Bologna 2002, 216.

${ }^{61}$ Cfr. Congregazione Delle CaUSe DeI SANTI, Normae servandae in inquisitionibus ab Episcopis faciendis in causis sanctorum, 7 febbraio 1983, AAS 75 (1983) I 396-403.

${ }^{62}$ P. MONETA, La giustizia..., cit., 216, nota 77.

${ }^{63}$ R. J. SARNO, Elementos preliminares en las causas de beatificación y canonización, in R. QUINTANA BEscós (ed.), Procesos de canonización. Comentarios a la Instrucción Sanctorum Mater, Ediciones San Dámaso, Madrid 2010, 36. 
sorta durante la vita del fedele e continuata, anzi aumentata col passare del tempo, presso la maggior parte del popolo di Dio.

Tornando alla legittimazione ad avviare la causa, essa esiste in capo a chiunque - individuo o associazione - nutra la convinzione che il Servo di Dio deceduto, il quale ha vissuto un'esistenza cristiana esemplare, si trova nella visione beatifica di Dio della Chiesa celeste e può intercedere per gli uomini ancora viatores nella storia: avviando una causa non si persegue un proprio interesse strettamente personale ma si coopera a «una funzione pubblica nella produzione dei modelli di santità ${ }^{64}$ imitabili e nella promozione del culto pubblico, costruendo un ponte tra la realtà misterica della Chiesa e quella istituzionale, tra Chiesa trionfante e Chiesa militante. Un'azione popolare, questa, non troppo 'vivisezionata' nei suoi profili giuridici dalla dottrina, forse perché reputati poco affascinanti e appetibili: eppure assai esplicativa di un tratto davvero ragguardevole della 'costituzione materiale' della Chiesa. Infatti in quell'ordito ben calibrato tra spinta dal basso e riscontro e validazione dall'alto, tra iniziativa dei fedeli nell'esercizio in specie dell'ufficio profetico e regale e risoluzione autoritativa della gerarchia campeggia in maniera evidente un carattere indelebile dell'ordinamento canonico: quello che vede i due 'poteri costituzionali' non antagonisti e rivali ma tra loro sinergicamente cospiranti. Una conspiratio che addiviene qui ad un 'verdetto' con cui la giustizia della Chiesa tocca il suo punto eccelso poiché «ardisce di farsi interprete dell'imperscrutabile giudizio del Creatore, rendendolo già concretamente percepibile in questo nostro mondo terreno e portando così a compimento quella continua tensione ad immedesimarsi nella stessa giustizia divina ${ }^{65}$. Non si tratta di una dichiarazione politica o ideologica, ma di un discernimento: «la Iglesia discierne lo que Dios está realizando en medio de la Iglesia misma y del mundo» ${ }^{66}$. E in tale discernimento il sensus del popolo di Dio non è in alcun modo rimpiazzabile o tacitabile: sia questo discernimento, per così dire, in positivo o in negativo.

Proprio su questo sfondo ci pare così assai significativo istituire una qualche rispondenza: non, bisogna ammetterlo, sul filo di un pedissequo rigor iuris, essendo palesemente del tutto atipico il processo di canonizzazione, sì da precludere frettolose simmetrie. Ma questo sbiadire ed anzi quest'eccedere l'ego-

${ }^{64}$ G. Dalla TORRe, Lezioni di diritto canonico, quinta edizione, G. Giappichelli Editore, Torino 2018, 286.

65 P. MONETA, La giustizia nella Chiesa, cit., 228.

${ }^{66}$ R. J. SARNO, Elementos preliminares en las causas de beatificación y canonización, cit., 36. 
centrica' spettanza individuale per proiettarsi, appunto 'superindividualmente', sulla lunghezza d'onda propriamente comunitaria di un 'giudizio' (che con la canonizzazione pontificia diverrà infallibile) condiviso, è un fermento giuridico che non può non rinvenire un bacino di coltura fertile in ogni segmento dello ius canonicum. Non può essere dunque un fuor d'opera concepire un interesse e una legittimazione alla caducazione di un atto - al fondo di cattivo discernimento - che nuoce a quel buon governo cui tutta la congregatio Ecclesiae ha diritto senza che vi sia, neppure invero prognosticamente (come fumus), un gravamen esclusivo di un fedele, il quale ultimo ne gode per sé proprio uti fidelis. Ovvero a goderne sarebbero i singuli uti coetus fidelium.

Insomma ci sentiamo di propugnare de iure condendo nella temperie attuale non tanto la positivizzazione di un diritto al buon governo, che già può pacificamente ammettersi tra i diritti e doveri cosiddetti fundamentales e che forse rimarrebbe sulla carta nonostante la sua parenetica ufficializzazione, come sovente avviene per le enunciazioni assiomatiche. Caldeggiamo piuttosto il riconoscimento esplicito di un diritto-dovere solo prima facie circoscritto e delimitato, essendo per contro suscettibile di essere realizzato oltreché assistito procedimentalmente in molteplici campi, scavalcando così con un colpo d'ala certe ineluttabili strettoie di istituti che qui abbiamo assunto in qualche modo in via euristica: segnatamente l'obligatio et ius christifidelium di affiancare l'autorità ecclesiastica nell'eliminazione di scorrettezze, irregolarità, sperequazioni e disparità di trattamento, ma pure strabismi, insipienze e distrazioni nel governo della Chiesa, in specie nella deputazione agli uffici; tutti 'accidenti', questi, che possono avere ripercussioni sulla salus animarum di più persone, se non altro cagionando quella bonae existimationis amissio vel aversio dei fedeli (parrocchiani) onesti e seri («probos et graves») che è menzionata dal Codice come causa di possibile rimozione del parroco (can. 1741 n. 3).

Quale ulteriore progresso nella formalizzazione tecnico-giuridica della natura ministeriale del governo ecclesiastico, dovrebbe pertanto riconoscersi il diritto dei fedeli di attivarsi in prima persona 'denunciando' la mancanza di idoneità dei titolari di uffici sia, come abbiamo sopra illustrato, durante l'approntamento e predeterminazione della provvista dell'ufficio, con un'opportuna 'incursione' nel procedimento amministrativo, sia una volta che essa sia avvenuta, quale 'protesta' ragionevole alla medesima: senza troppo insistere peraltro su tale biforcazione - apparente, essendo l'una compensazione delle disfunzioni dell'altra - che andrebbe fatta convergere verso un unico intento. Un intervento comunque sempre, beninteso, sia a monte sia a valle, azionato 
con quel garbo, tatto e discrezione che sono la bussola senza la quale sovente l'uso di un diritto può tramutarsi in arrogante soperchieria e sopraffazione; tanto più in Ecclesia. Si staglia, oltre al can. 209 e alla doverosa tutela della communio, il can. $223 \$ 1$ a circoscrivere le frontiere oltrepassando le quali, e dunque intaccando il bene comune della Chiesa o i diritti altrui, l'esercizio di un diritto diventa prevaricazione. Segnatamente «los fieles abusarían del derecho de manifestar a los pastores opiniones relativas al bien de la Iglesia cuando no se salvara la integridad de la fe y las costumbres, o cuando se hiciera sin reverencia o sin tener en cuenta la utilidad común y la dignidad de las personas» ${ }^{67}$; e, ancora, «aunque podría ser moralmente lícito descubrir defectos, pecados o delitos ajenos por necesidades de un bien superior, ese derecho se convertiría en abusivo cuando se lesionara ilegítimamente la buena fama o se violara el derecho de las personas a proteger su propria intimidad» ${ }^{68}$. Ma, sia pur opportunamente 'recintato', mai forse come oggi è manifesta l'opportunità di tale intervento dei fedeli che si ponga quale 'resistenza' propositiva ad atti di governo 'inaccettabili' e 'insostenibili' ${ }^{69}$ : e per il suo esercizio la canonistica deve senza indugio preordinare strumenti giuridici appropriati. Per questa via, attraverso un misurato concorso popolare, oltre a smascherare episodi di «carrierismo, arrampicatori, nepotismo»o di «simonia educata ${ }^{70}$ nella Chiesa, contro i quali papa Francesco tuona veementemente quasi ogni giorno, si otterrebbe certamente di mitigare se non di cancellare quella lontananza, indifferenza ed apatia che sovente i fedeli mostrano per le attività ecclesiali che non li concernono immediatamente. Tra l'altro sarebbe oltremodo opportuno che la critica di una scelta e la sua sostituzione «non procedesse al traino o all'ombra delle istanze esterne (e spesso ostili) alla Chiesa, ma come uno sviluppo o maturazione endogena della compagine salvifica» ${ }^{71}$ che si fa fraternamente $\mathrm{e}$ lodevolmente carico di rimediare alle mancanze interne e al contempo precorre e sventa inframmettenze 'nemiche'.

Si è rimembrato che «Nella Chiesa delle origini e nei primi secoli [...] il livello di partecipazione e coinvolgimento dei christifideles era molto intenso e

67 J. L. Llaquet, Abuso de derecho, in J. Otaduy - A. Viana - J. Sedano (eds.), Diccionario general de derecho canónico, I, Thomson Reuters Aranzadi, Cizur Menor (Navarra) 2012, 94.

${ }^{68}$ J. L. Llaquet, Abuso..., cit., 94.

${ }^{69}$ Cfr. le riflessioni di P. GHERRI, Petitio..., cit., 339-340.

${ }^{70}$ FranCESCO, Il vero potere è servizio, omelia durante la messa nella cappella della Domus Sanctae Marthae di martedì 21 maggio 2013 (cfr. www.vatican.va).

${ }^{71}$ M. Del Pozzo, Lo statuto giuridico..., cit., 163. 
sentito. Il progressivo allontanamento del popolo dalla vita della comunità non sembra allora un sintomo troppo confortante e incoraggiante» ${ }^{72}$. Invertire questa tendenza - che è insieme, al fondo, restaurare l'originario paradigma evangelico del servizio nel governo - anche con il varo di un nuovo diritto dei fedeli - sia singolarmente sia riuniti in aggregazioni, sodalizi, movimenti - che li interpelli in un tornante così delicato come quello relativo alla designazione agli incarichi non rappresenterebbe un istigare l'occhiuta sorveglianza dei 'sudditi' nei confronti delle autorità quasi per riscattare polemicamente secoli di subalterna minorità e muta sottomissione, o un porgere l'appiglio ad attacchi gratuiti alle autorità. Invece si prefiggerebbe in prima battuta un fine poderosamente educativo: quello di rendere sempre più interiormente 'familiare', agapica ed esteriormente visibile la compartecipazione di tutte le componenti della Chiesa alla sua missione anche kerigmatica e altresì allo stesso potere nella medesima. Un diritto, quello prefigurato, che inoltre, secondo la logica aletica permeante la comunione, non può non includere l'aspetto deontico dell'obbligo, secondo l'endiadi 'dovere-diritto' (in quest'ordine del resto nel Codice) così gravida di valenze nell'ordinamento canonico. Anche per questo la sua 'consacrazione', al di là dell'utilizzo pratico e quindi dalla numerosità delle 'denunce', rivestirebbe una portata sistematica e una carica propulsiva non dappoco. D'altronde già oltre un decennio fa ci si rammaricava che «le dichiarazioni sull'abilitazione dei fedeli»-anche di «chi, pur non essendo destinatario diretto dell'atto, sia comunque interessato alla materia ${ }^{73}-<$ a interloquire e incidere sull'azione della gerarchia costituiscono in realtà enunciazioni teoriche abbastanza generiche che richiederebbero, per essere davvero efficaci e imporsi nell'esercizio concreto delle funzioni di giurisdizione, di essere precisate in puntuali previsioni della legislazione positiva. /I codici, tuttavia, non contengono alcuna disposizione che promuova la partecipazione dell'unusquisque de populo» ${ }^{74}$ : forse è giunta l'ora, e la riforma in corso della Curia romana sotto il segno dell'"estroversione ${ }^{75}$, potrebbe rappresentare il primo banco di prova e costituire una molla davvero vigorosa.

${ }^{72}$ M. Del Pozzo, Lo statuto giuridico..., cit., 137.

73 I. Zuanazzi, Praesis ut prosis. La funzione amministrativa nella diakonía della Chiesa, Jovene, Napoli $2005,618$.

74 I. ZuANAZZI, Praesis ut prosis..., cit., 618-619.

75 Cfr. Francesco, Discorso per la presentazione degli auguri natalizi della Curia romana, 21 dicembre 2017, L'osservatore romano 22 dicembre 2017 4-5. 
Uno dei 'vessilli' del sommo pontefice regnante è infatti quello della sinodalità - termine impostosi dopo il Concilio e che attualmente ha varcato ed è fuoriuscito dai contorni primigeni-: un principio che sinora parrebbe essere stato esiliato in una sorta di «dormizione giuridica» proprio per «la mancata attivazione di strumenti concreti capaci di sostenere un ruolo di attiva responsabilità da parte del popolo di Dio nel governo delle questioni relative alla vita della Chiesa» ${ }^{76}$. Lo stesso papa ha richiamato il celeberrimo principio, «caro alla Chiesa del primo millennio» quod omnes tangit ab omnibus tractari de$b e t^{77}$ a raffigurare l'appello a tutto il popolo di Dio, 'finalmente' riposizionato nella sua centralità e unitarietà, a farsi udire dai pastori per la salute dell'intero organismo ecclesiale. Ciò comporta non una confusa e caotica intromissione, irriflessa e anarchica, nei processi decisionali, restando la risoluzione finale saldamente imputata all'ufficio capitale: ma consentire - senza intonazioni di 'concessione graziosa' - e pungolare chi ha argomenti pertinenti ad esporli con un atto supererogatorio tipicamente cristiano per il bene comune non può non essere da una parte, ecclesiologicamente, nitida icona di cattolicità e dall'altra, pragmaticamente, veicolo del diritto al buon governo e al contempo guarentigia affinché esso si realizzi.

\section{QUALCHE RIFLESSIONE SULLA DENUNTIATIO CHRISTIFIDELIUM IN ORDINE ALLE FATTISPECIE DI CUI AL CAN. 1389 E IN ALTRI CASI. UNA 'DIREZIONE DI MARCIA' DESUMIBILE DA ALCUNI PROVVEDIMENTI}

Nella monografia dedicata a questo argomento abbiamo dilatato l'orizzonte sino ad occuparci anche della denuncia penale vera e propria, poiché la nomina o la permanenza di persona inidonea potrebbe integrare la perpetrazione di un delitto 'proprio' da parte di colui che deve provvedere alla designazione o al controllo ex can. 1389. I casi di abuso o sviamento, latamente inteso, del potere da parte dell'autorità possono incidere variamente nella provvisione degli uffici ecclesiastici: così l'autorità potrebbe «usare la discrezionalità nella provvista per allontanare una persona non gradita all'autorità, trasferire una persona per rendere inattuabile la ricerca di una verità che possa essere scomo-

${ }^{76}$ F. S. REA, Interessi diffusi e ordinamento canonico. Le posizioni giuridiche meta-individuali tra assorbimento del Civis nelle formazioni sociali e Spannung ontologico del Christifidelis nella realtà comunitaria della Chiesa, Turisa editrice, Acerra (NA) 2018, 650.

77 Francesco, Discorso per la commemorazione del $50^{\circ}$ anniversario dell'istituzione del Sinodo dei Vesco$v i, 17$ ottobre 2015 (cfr. www.vatican.va). 
da, la provvista (equivalente ad una rimozione) effettuata come risposta a pressioni di una parte della comunità o persino di una autorità terza senza il dovuto fondamento, la fenomenologia più o meno celata di promuovere per rimuovere che colloca persone inidonee in posti di responsabilità, la precarizzazione delle nomine per tenere sotto controllo i ministri, la nomina di una persona ad una molteplicità di uffici di impossibile adempimento per coprire le apparenze, il rendere inutile un ufficio per il fatto di attribuirne la titolarità ad una certa persona con la sola finalità di garantire il suo sostentamento economico, ecc. ${ }^{78}$. Come si constata, sovente in primo piano è proprio l'idoneità del titolare che risulta difettosa. Solo i casi più gravi costituiranno delitto, eventualmente con l'accordo fraudolento o la correità del titolare dell'ufficio.

$\mathrm{Ci}$ si può quindi domandare se il fedele che ne sospetta fortemente la commissione, avendone non lievi indizi o addirittura prove inequivocabili (anche se ciò sarà sovente non semplice) abbia non solo il diritto ma anche l'obbligo di denunciarlo all'autorità ecclesiastica. Rinviando alla nostra trattazione più estesa ${ }^{79}$, ora solo rimembriamo come da una lettura in parallelo della vigente codificazione con quella abrogata, da un esame delle opinioni dottrinali, da una ricognizione di alcuni interventi normativi extra codiciali che sollecitano il popolo di Dio a collaborare nella prevenzione e sanzione (disciplinare o penale) di certi comportamenti (l'Istruzione della Congregazione per il culto divino e la disciplina dei sacramenti Redemptionis sacramentum del 25 marzo 2004, per le segnalazioni di abusi in materia liturgica ${ }^{80}$, ovvero la normativa relativa ai delicta graviora ${ }^{81}$ ) oppure denotano una rafforzata intransigenza nel chiedere conto delle proprie responsabilità anche ai titolari degli uffici apicali (il Motu Proprio Come una madre amorevole del 4 giugno $2016^{82}$ che prevede la rimozione del vescovo negligente), nonché infine dai reiterati appelli recenti del papa e dell'episcopato perveniamo alla conclusione che si possa scorgere una 'direzione dei marcia'. Proprio in questa prospettiva pensiamo

\footnotetext{
78 F. PUIG, Governo e discrezionalità..., cit., 74.

79 Cfr. di nuovo G. BonI, Il buon governo..., cit., 175 ss.

80 Cfr. Congregazione Per Il CUlto Divino E la Disciplina DeI SACRAMENTi, Redemptionis sacramentum su alcune cose che si devono osservare ed evitare circa la Santissima Eucaristia, 25 marzo 2004, AAS 96 (2004) 549-601, in particolare nn. 183-184.

81 Cfr. Congregazione Per la dotTrina della fede, Normae de delictis Congregationi pro doctrina fidei reservatis seu Normae de delictis contra fidem necnon de gravioribus delictis, 21 maggio 2010, AAS 102 (2010) 419-434.

82 Cfr. Francesco, Lettera Apostolica Motu Proprio Come una madre amorevole, 4 giugno 2016, AAS 108 (2016) 715-717.
} 
che sarebbe auspicabile - ponderando i pro e i contro - una formalizzazione nel diritto vigente di una norma analoga a quella cassata del Codex pianobenedettino ${ }^{83}$, contemplando espressamente la denuncia (non l'obbligo giuridico di denuncia) dei fedeli quale canale della notitia criminis, sia pur opportunamente cinta da garanzie (severità della punizione della denuncia calunniosa, oltre al presidio granitico dello ius defensionis del denunciato) in funzione dissuasiva rispetto ad iniziative inconsulte e temerarie. D'altro canto, valorizzare la denuntiatio christifidelium «trova le sue radici ultime nello stesso Vangelo (cfr. Mt 18,15-17), che nel descrivere l'iter sanzionatorio nella Chiesa evidenza molto chiaramente l'iniziativa del fedele» ${ }^{84}$ : si sarebbe dinanzi ad una rilevante epifania della collaborazione christifidelium al munus regendi.

$\mathrm{E}$ questa denuncia penale dovrebbe in qualche modo correlarsi, nella distinzione, certo, ma pure nella correlazione, con quella più generica di cui sopra abbiamo proposto la positivazione in rapporto alla mancanza di idoneità agli uffici: i piani sono differenti ma non per questo del tutto scollati, separati e non comunicanti. La denuncia dovrebbe essere oculatamente 'agevolata', senza ovviamente trascendere in inviti gridati e indiscriminati alla medesima, tanto demagogici quanto fuorvianti. Si dovrebbe al contrario istillare nei fedeli l'idea che, al di là del vocabolo 'denuncia' il quale può risvegliare tristi e inquietanti 'scenari inquisitoriali', tale informativa è animata da un fine altamente pastorale, colorandosi in tal modo delle sfumature della solidarietà alla missione della Chiesa. Si fa leva sulla sincera affezione per la Chiesa che induce i fedeli a deflettere da ogni reticenza e passività per prodigarsi in prima persona dinanzi alla tragica miseria o anche alla sconsideratezza del comportamento di alcuni. Non si tratta di resuscitare nuove fanatiche 'cacce alle streghe' o di sobillare mormorazioni oppure un parossistico incrementarsi di esposti e doglianze. Ma, come ha recentemente quasi implorato papa Bergoglio, «è necessario che ciascun battezzato si senta coinvolto nella trasformazione ecclesiale e sociale di cui tanto abbiamo bisogno», e «Tale solidarietà ci chiede [...] di denunciare tutto ciò che possa mettere in pericolo l'integrità di qualsiasi persona» ${ }^{85}$.

Potrebbe poi accadere che l'ordinario, benché non possa mai sbrigativamente sprezzare o snobbare la denuntiatio dando invece ascolto ai fedeli, ac-

Si trattava del can. 1935.

${ }^{84}$ C. J. ERrázuriz M., Il "munus docendi Ecclesiae": diritti e doveri dei fedeli, Giuffrè Editore, Milano $1991,55$.

85 FrANCESCO, Lettera al popolo di Dio, 20 agosto 2018 (cfr. www.vatican.va). 
certi però che quanto riferito non è penalmente rilevante, ovvero non rientra nelle condotte delittuose tracciate dal sopradetto canone, o ancora comunque preferisca, nonostante una notitia criminis verisimilis, desistere dall'inquisire, rientrando questo nella sua autonomia decisionale. Ma in tutte queste evenienze, pur in assenza di castigo penale per mancanza di presupposti, l'ordinario potrebbe tuttavia prendere in considerazione di rettificare la provvista inopportuna compiendo i passi necessari per una sua sostituzione. La denuncia giudiziale si potrebbe mutare in denuncia 'canonica' a seguito di qualche abuso nella disciplina, richiamando il vescovo al suo dovere di vigilanza (can. $392 \$ 2$ ). Emergono anche qui quelle sovrapposizioni tra diritto, sanzioni, azioni e procedure penali e diritto, sanzioni, azioni e procedure disciplinari ovvero amministrative già segnalate e sulle quali in questa sede non possiamo che rimandare alla letteratura specifica ${ }^{86}$. Si chiude così il circolo con quanto abbiamo osservato in precedenza, evidenziandosi ancora una volta la crucialità dell'apporto - in fondo sempre alla verità - dei singoli christifideles, integrante e complementare rispetto alla ministerialità sacra e all'assetto giurisdizionale; quell'organica cooperatio di tutti i membri del popolo di Dio che stenta forse ancora a radicarsi e concretizzarsi «nella forma mentis e nell'agire del clero e del laicato» ${ }^{87}$ : e che va invece assecondata e supportata.

In questa circostanza, così come in caso di proposizione di semplice segnalazione, di una petitio o di una remonstratio, ovvero dell'avvio di un ricorso amministrativo, non si sarebbe affatto dinanzi ad una spaccatura o lacerazione all'interno della comunità, né ad un insulto o affronto all'autorità, tutt'altro. Rivolgersi all'autorità, denunciando, dimostra fiducia che essa affronterà il problema di illegittimità e ingiustizia e lo risolverà: da parte del cristiano non è atto di indocilità, di ribellione, e tanto meno di eversione, ma azione nella e per la Chiesa. Significa anzitutto la maturazione di un'intima e ben meditata coscienza della propria corresponsabilità, avere in qualche modo cognizione e signoria degli strumenti messi a disposizione dal diritto canonico nonché confidenza speranzosa che l'autorità non lascerà cadere nel vuoto legittime aspettative: ciò può iniettare un antidoto davvero portentoso alle spinte centrifughe, alla disaffezione e allo straniamento, ma anche al dissenso prevenuto e acrimonioso, potendo creare paradossalmente un babitat più 'domestico' di condivisione tra

${ }^{86}$ Cfr. recentemente G. P. MonTINI, Il diritto disciplinare canonico, Quaderni di diritto ecclesiale 31 (2018) 264-278.

87 M. Del Pozzo, Lo statuto giuridico..., cit., 140. 
fedeli e pastori. Si tratta veramente di evincere tutti i riverberi concreti dalle scaturigini assiologiche del potere genuinamente diaconale (potestas functio) e dello status di christifidelis cooperante ad aedificationem corporis Christi anche riguardo al regimen Ecclesiae e proprio al munus administrandi, in una dialettica che non è mai scontro e collisione ma sempre complementarietà.

\section{DigNUS EST!}

Osserviamo conclusivamente come recentemente si sia riferito con ammirazione di una cerimonia assai suggestiva che si svolge in occasione delle ordinazioni presbiterali nella Chiesa greco cattolica di Oradea in Romania: alla domanda del vescovo "Ne è degno?", coralmente tutta l'assemblea risponde, in canto, ripetendo più volte "Vrednic este!", "ne è degno"; si tratta, si è commentato, di «Una parola "liturgica" che bene esprime il coinvolgimento della comunità cristiana, una parola cui si può giungere dopo che un serio percorso di conoscenza è stato attuato ${ }^{88}$. Tale cerimonia, invero, non è per nulla una novità, riproducendo la prassi, insediatasi nel primo millennio della Chiesa, di sollecitare la consultazione e il suffragio del popolo cristiano non solo per la consacrazione di un vescovo, ma per ogni ammissione agli ordini maggiori, presentando gli ordinandi ai fedeli e spronando con impeto a far sapere se vi fosse qualcosa in contrario ${ }^{89}$; spesso non ci si accontentava del silenzio - che poteva essere rassegnazione obtorto collo quasi ad un giogo oppressivo -, ma si invitava ad approvare appunto acclamando 'dignus est' ${ }^{90}$. E per lungo tempo è stato così.

La lex orandi e la lex credendi non possono mai essere disgiunte dalla lex agendi: anche se può apparire curioso, l'enfatizzazione del diritto dei fedeli a denunciare la mancanza di idoneità dei titolari degli uffici ecclesiastici e quindi ad avere una parte non modesta nel processo di deliberazione/cooptazione ecclesiale sarebbe un ulteriore 'transito' in quel 'tirocinio' nella recezione del Concilio Vaticano II non ancora ultimato. Con un piccolo tassello aggiunto al corpus normativo canonico si metterebbe in pratica un tratto non secondario di quella pastoralità del governo ecclesiale davvero permeabile non tanto alla

\footnotetext{
88 A. MigliavaCCA, Irregolarità e impedimenti..., cit., 443.

89 Cfr. la sintesi di M. GraTtier, Bans d'ordination, Dictionnaire de droit canonique, II, Libraire Letouzey et Ané, Paris 1937, 96.

90 Cfr. M. Grattier, Bans..., cit., 96.
} 
collaborazione ma a quella piena corresponsabilità, ad intra e ad extra, di tutti i fedeli - ognuno con i suoi insostituibili 'doni' - tanto decantata da numerosi documenti magisteriali ma, per alcuni versanti, ancora segregata in un onirico iperuranio: come se si temesse di trovarsi in balia di capricciose e volubili iniziative dei singoli che invece possono costituire, oggi più che mai, 'valvole di sicurezza' contro defecta cognoscentiae vel iudicii, fidandosi del "fiuto" del popolo di Dio ${ }^{91}$. Tale diritto, da una parte, affonderebbe le radici in quella diaconalità dei munera ecclesiali secondo cui il titolare di funzioni pubbliche non è dominus ma fidelis dispensator; dall'altra, sconfessando ogni 'clericalismo' - come papa Francesco non si stanca di rimproverare - recupererebbe un legame fecondo tra chi detiene la leadership, se così la vogliamo definire, e i semplici battezzati - prima ancora che nelle norme, nella vita della comunità cristiana - che si è allentato e che per converso è linfa vitale inalveante la dignitas et libertas filiorum Dei nella communio gratiae et amoris. Si profila una sfida, anzi un appuntamento imperdibile per l'autorità gerarchica e per tutti i fedeli in una Chiesa in cui, «come in una piramide capovolta il vertice si trova al di sotto della base ${ }^{92}$ : sempre con la coscienza che «l'obiettivo di questi processi partecipativi non sarà principalmente l'organizzazione ecclesiale, bensì il sogno missionario di arrivare a tutti» ${ }^{93}$, cioè il compimento coeso dell'opera evangelizzatrice della Chiesa nella giustizia.

${ }^{91}$ Cfr. Francesco, Discorso ai partecipanti al Convegno per i nuovi vescovi promosso dalla Congregazione per $i$ vescovi e dalla Congregazione per le Chiese orientali, 19 settembre 2013 (cfr. www.vatican.va).

92 FRANCESCO, Discorso per la commemorazione del $50^{\circ}$ anniversario dell'istituzione del Sinodo dei Vescovi, 17 ottobre 2015, cit.

93 FRANCESCO, Esortazione Apostolica Evangelii gaudium sull'annuncio del Vangelo nel mondo attuale, 24 novembre 2013, n. 31 (cfr. www.vatican.va). 


\section{Bibliografía}

ARRIETA, J. I., Funzione pubblica e attività di governo nell'organizzazione centrale della Chiesa: il Regolamento generale della Curia romana, Ius Ecclesiae 4 (1992) 585-613.

BAURA, E., Analisi del sistema canonico di giustizia amministrativa, in E. BAURA J. CANOSA (eds.), La giustizia nell'attività amministrativa della Chiesa: il contenzioso amministrativo, Giuffrè Editore, Milano 2006, 1-58.

—, Il "buon governo": diritti e doveri dei fedeli e dei pastori, in GRUPPO ITALIANO DOCENTI DI DiRITTO CANONICO (a cura di), Il governo nel servizio della comunione ecclesiale, Glossa, Milano 2017, 3-30.

BONI, G., Il buon governo nella Chiesa. Inidoneità agli uffici e denuncia dei fedeli, Mucchi Editore, Modena 2019.

CARIDÀ, R., Codice di comportamento dei dipendenti pubblici e principi costituziona$l i$, federalismi.it. Rivista di diritto pubblico italiano, comparato, europeo 14 (2016) n. 25 1-28.

CARLONI, E., Il nuovo codice di comportamento e il rafforzamento dell'imparzialità dei funzionari pubblici, Istituzioni del federalismo 33 (2013) 377-407.

CASSESE, S., «Maladministration» e rimedi, Il foro italiano 127 (1992) V 243250.

CaVAllo Perin, R., L'etica pubblica come contenuto di un diritto degli amministrati alla correttezza dei funzionari, in F. MERLONI - R. CAVALlo PERIN (eds.), Al servizio della Nazione. Etica e statuto dei funzionari pubblici, Codice Edizioni, Milano 2009, 147-161.

Cerulli Irelli, V., Per una politica dell'etica pubblica nella disciplina delle funzioni amministrative, Astrid 14/2019, www.astrid-online.it 1-24.

Clarich, M. - Mattarella, B. G., La prevenzione della corruzione, in B. G. Mattarella - M. Pelissero (eds.), La legge anticorruzione. Prevenzione e repressione della corruzione, G. Giappichelli Editore, Torino 2013, 59-69.

Dalla Torre, G., Lezioni di diritto canonico, quinta edizione, G. Giappichelli Editore, Torino 2018.

Del Portillo, Á., Laici e fedeli nella Chiesa. Le basi dei loro statuti giuridici, Giuffrè Editore, Milano 1999 (prima edizione Fieles y laicos en la Iglesia. Bases de sus respectivos estatutos jurídicos, Eunsa, Pamplona 1981).

Del Pozzo, M., Lo statuto giuridico fondamentale del fedele, Edusc, Roma 2018.

ERRÁZuriz M., C. J., Il "munus docendi Ecclesiae": diritti e doveri dei fedeli, Giuffrè Editore, Milano 1991. 
GHERRI, P., L'autotutela amministrativa come supplemento di conoscenza: la remonstratio canonica (Cann. 1732-1734 CIC), in IDEM (ed.), Decidere e giudicare nella Chiesa. Atti della VI giornata canonistica interdisciplinare, Lateran University Press, Città del Vaticano 2012, 315-384.

- Petitio, remonstratio, exceptio. Cenni esplorativi sui modi di non-esecuzione degli atti amministrativi singolari, Ius Ecclesiae 27 (2015) 339-356.

González del Valle, J. Ma , Sub can. 1043, in Á. MarzoA - J. Miras R. Rodríguez-OcaÑa (eds.), Comentario exegético al Código de Derecho Canónico, III/1, tercera edición actualizada, Eunsa, Pamplona 2002, 989-990.

Grattier, M., Bans d'ordination, Dictionnaire de droit canonique, II, Libraire Letouzey et Ané, Paris 1937, 96-97.

Herranz, J., De principio legalitatis in exercicio potestatis ecclesiasticae, in PONTIficia Commissio Codici Iuris Canonici Recognoscendo (ed.), Acta Conventus Internationalis Canonistarum Romae diebus 20-25 maii 1968 celebrati, Typis Polyglottis Vaticanis, Città del Vaticano 1970, 221-238.

Hervada, J. - Lombardía, P., El derecho del pueblo de Dios. Hacia un sistema de derecho canónico, I, Introducción. La constitución de la Iglesia, Universidad de Navarra, Pamplona 1970.

Hervada, J., Elementos de derecho constitucional canónico, Eunsa, Pamplona 1987.

-, Diritto costituzionale canonico, prefazione all'edizione italiana di G. LO Castro, Giuffrè Editore, Milano 1989.

LABANDEIRA, E., Il ricorso gerarchico canonico: «petitum»e «causa petendi», Ius Ecclesiae 3 (1991) 103-118.

Llaquet, J. L., Abuso de derecho, in J. Otaduy - A. Viana - J. Sedano (eds.), Diccionario general de derecho canónico, I, Thomson Reuters Aranzadi, Cizur Menor (Navarra) 2012, 93-94.

Llobell, J., I processi matrimoniali nella Chiesa, Edusc, Roma 2015.

Mattarella, B. G., Le regole dell'onestà. Etica, politica, amministrazione, il Mulino, Bologna 2007.

MATteInI, P. - ORsinI, L., Il procedimento disciplinare dei dipendenti pubblici fra modifiche legislative e giurisprudenza della Corte di Cassazione 2015/2016 Aran, Occasional paper 6/2016, http://www.arangenzia.it/index.php/statistiche-e-pubblicazioni/ 1-50.

Miele, M., Ancora sul promotore di giustizia, JusOnline 2/2018 63-79.

MigliavacCa, A., Idoneidad para oficios y ministerios, in J. OTAdUY - A. Viana - J. Sedano (eds.), Diccionario general de derecho canónico, IV, Thomson Reuters Aranzadi, Cizur Menor (Navarra) 2012, 361-365. 
—, Irregolarità e impedimenti: vie di conoscenza e di verifica al servizio del discernimento, Quaderni di diritto ecclesiale 28 (2015) 423-443.

Miras, J., Sub can. 50, in Á. MarzoA - J. Miras - R. Rodríguez-Ocaña (eds.), Comentario exegético al Código de Derecho Canónico, I, tercera edición actualizada, Eunsa, Pamplona 2002, 557-562.

Miras, J. - Canosa, J. - Baura, E., Compendio di diritto amministrativo canonico, Edusc, Roma 2007.

MONETA, P., I soggetti nel giudizio amministrativo ecclesiastico, in La giustizia amministrativa nella Chiesa, Libreria Editrice Vaticana, Città del Vaticano 1991, 55-70.

—, La giustizia nella Chiesa, nuova edizione, il Mulino, Bologna 2002.

-, Introduzione al diritto canonico, quarta edizione, G. Giappichelli Editore, Torino 2016.

Montini, G. P., Il diritto disciplinare canonico, Quaderni di diritto ecclesiale 31 (2018) 264-278.

PUIG, F., Governo e discrezionalità nella collazione di uffici ecclesiastici, Ius Ecclesiae 30 (2018) 55-78.

RaCCA, G. M., Disciplina e onore nell'attuazione costituzionale dei codici di comportamento, in F. MERLONI - R. CAVAllo PERIN (eds.), Al servizio della Nazione. Etica e statuto dei funzionari pubblici, Codice Edizioni, Milano 2009, 250-265.

REA, F. S., Interessi diffusi e ordinamento canonico. Le posizioni giuridiche metaindividuali tra assorbimento del Civis nelle formazioni sociali e Spannung ontologico del Christifidelis nella realtà comunitaria della Chiesa, Turisa editrice, Acerra (NA) 2018.

Rivella, M., Il diritto disciplinare della Curia Romana, Quaderni di diritto ecclesiale 31 (2018) 320-328.

Rodríguez-OCAÑA, R., Sub can. 1674, in Á. MarzOA - J. Miras - R. RodrígueZ-OcaÑa (eds.), Comentario exegético al Código de Derecho Canónico, IV/2, tercera edición actualizada, Eunsa, Pamplona 2002, 1849-1865.

SANDUlli, A. M., Manuale di diritto amministrativo, Jovene, Napoli 1980.

SARNO, R. J., Elementos preliminares en las causas de beatificación y canonización, in R. Quintana Bescós (ed.), Procesos de canonización. Comentarios a la Instrucción Sanctorum Mater, Ediciones San Dámaso, Madrid 2010, 27-52.

Schouppe, J.-P., Diritti fondamentali dei fedeli in rapporto alla partecipazione al governo dei beni temporali, Ius Ecclesiae 26 (2014) 397-414.

Tognoni, G., La tutela degli interessi diffusi nell'ordinamento canonico, Quaderni di diritto ecclesiale 8 (1995) 321-344. 
Viana, A., Aspectos de la relación del fiel con la organización eclesiástica, Fidelium iura 4 (1994) 79-106.

—, La comprobación de la idoneidad para el oficio eclesiástico y el orden sagrado, Ius Ecclesiae 28 (2016) 345-366.

VILADRICH, P., La declaración de derechos y deberes de los fieles (Cánones 10 al 30), in REDACCIÓN IUS CANONICUM (ed.), El proyecto de Ley fundamental de la Iglesia. Texto y análisis crítico, Eunsa, Pamplona 1971, 123-159.

ZuANAZZI, I., La legittimazione a ricorrere uti fidelis per la tutela di diritti comunitari, in R. Bertolino - S. Gherro - G. Lo Castro (eds.), Diritto 'per valori' e ordinamento costituzionale della Chiesa, G. Giappichelli Editore, Torino 1996, 399-450.

—, Praesis ut prosis. La funzione amministrativa nella diakonía della Chiesa, Jovene, Napoli 2005.

—, La corresponsabilità dei fedeli laici nel governo ecclesiale, in GRUPPO ITALIANO Docenti Di DiriTto CANONICO (a cura di), Il governo nel servizio della comunione ecclesiale, Il "buon governo": diritti e doveri dei fedeli e dei pastori, Glossa, Milano 2017, 101-148. 